\title{
Shear Wave Seismic Study Comparing 9C3D SV and SH Images with 3C3D C-Wave Images
}

\section{FINAL REPORT}

\author{
Reporting Period Start Date: January 1, 2003
}

Reporting Period End Date: June 30, 2004

John Beecherl, Principal Investigator*

Bob A. Hardage**

July 2004

Work performed under DOE Grant DE-FG26-03NT15431

\author{
Prepared by \\ *Vecta Technology \\ 5950 Cedar Springs Road, Suite 225 \\ Dallas, TX 75235 \\ and \\ **Bureau of Economic Geology \\ John A. and Katherine G. Jackson School of Geosciences \\ The University of Texas at Austin \\ University Station, P.O. Box X \\ Austin, TX 78713-8924
}




\section{DISCLAIMER}

This report was prepared as an account of work sponsored by an agency of the United States Government. Neither the United States Government nor any agency thereof, nor any of their employees, makes any warranty, express or implied, or assumes any legal liability for responsibility for the accuracy, completeness, or usefulness of any information, apparatus, product, or process disclosed, or represents that its use would not infringe privately owned rights. Reference herein to any specific commercial product, process, or service by trade name, trademark, manufacturer, or otherwise does not necessarily constitute or imply its endorsement, recommendation, or favoring by the United States Government or any agency thereof. The views and opinions of authors expressed herein do not necessarily state or reflect those of the United States Government or any agency thereof. 


\begin{abstract}
The objective of this study was to compare the relative merits of shear-wave (Swave) seismic data acquired with nine-component (9-C) technology and with threecomponent (3-C) technology. The original proposal was written as if the investigation would be restricted to a single 9-C seismic survey in southwest Kansas (the Ashland survey), on the basis of the assumption that both 9-C and 3-C S-wave images could be created from that one data set. The Ashland survey was designed as a 9-C seismic program. We found that although the acquisition geometry was adequate for 9-C data analysis, the source-receiver geometry did not allow 3-C data to be extracted on an equitable and competitive basis with 9-C data. To do a fair assessment of the relative value of 9-C and 3-C seismic S-wave data, we expanded the study beyond the Ashland survey and included multicomponent seismic data from surveys done in a variety of basins. These additional data were made available through the Bureau of Economic Geology, our research subcontractor.

Bureau scientists have added theoretical analyses to this report that provide valuable insights into several key distinctions between $9-\mathrm{C}$ and 3-C seismic data. These theoretical considerations about distinctions between 3-C and 9-C S-wave data are presented first, followed by a discussion of differences between processing 9-C commonmidpoint data and 3-C common-conversion-point data. Examples of 9-C and 3-C data are illustrated and discussed in the last part of the report.

The key findings of this study are that each S-wave mode (SH-SH, SV-SV, or PSV) involves a different subsurface illumination pattern and a different reflectivity behavior and that each mode senses a different Earth fabric along its propagation path because of the unique orientation of its particle-displacement vector. As a result of the distinct orientation of each mode's particle-displacement vector, one mode may react to a critical geologic condition in a more optimal way than do the other modes. A conclusion of the study is that 9-C seismic data contain more rock and fluid information and more sequence and facies information than do 3-C seismic data; 9-C data should therefore be acquired in multicomponent seismic programs whenever possible.
\end{abstract}




\section{CONTENTS}

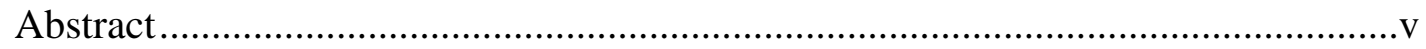

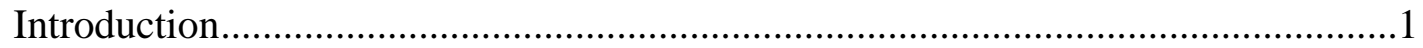

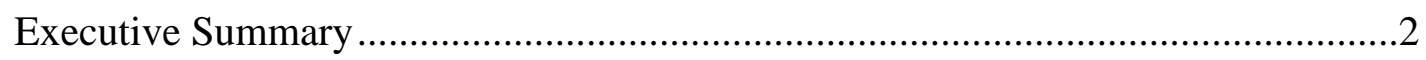

Basic Concepts That Distinguish 9-C and 3-C S-Wave Data........................................

Concept 1: Vector-Based Technology ……………………....................................

Concept 2: Components of Multicomponent Seismic Data...................................10

Argument 1: Sensing the Earth Fabric...................................................................12

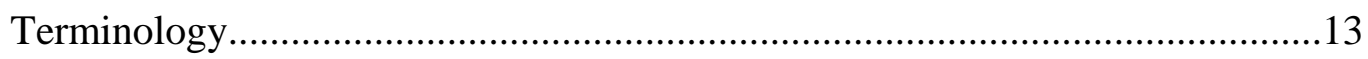

Marine Environments ...................................................................................

Argument 2: Multicomponent Reflectivities .......................................................17

Argument 3: Multicomponent Illumination.........................................................26

Elastic-Wavefield Seismic Stratigraphy ……………………………...................33

Data-Processing Differences between 9-C and 3-C S-Wave Data .............................36

Common-Midpoint (CMP) Imaging ...................................................................36

Common-Conversion-Point (CCP) Imaging.........................................................37

CMP and CCP Velocity Analyses ......................................................................40

CMP and CCP Stacking ..................................................................................43

Examples of 9-C and 3-C S-Wave Data ...............................................................49

Distinctions between SH-SH and SV-SV S-Wave Modes .....................................50

3-C Data Polarizations ......................................................................................

Making 3-C and 9-C Images Depth Equivalent.....................................................61 
Conclusions.

References.

Figures

1. Distinction between vector and scalar seismic sources .5

2. An example of three orthogonal seismic vector sources working to produce ninecomponent vertical seismic profile data .7

3. Twelve vector sources ready to deploy across a large 9C3D seismic survey .................7

4. Standard three-component moving-coil geophone ...............................................8

5. Micro-Electro-Mechanical System three-component seismic sensor available from

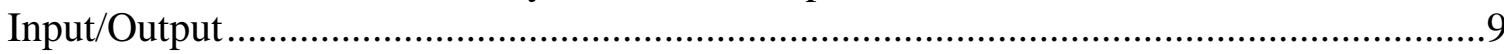

6. MEMS three-component sensor package available from Sercel ................................10

7. Full-elastic, multicomponent seismic wavefield propagating in a homogeneous Earth consisting of a compressional mode $\mathrm{P}$ and two shear modes, SV and SH ......................11

8. Distinction between SH and SV shear wave displacements ....................................12

9. Options for acquiring multicomponent seismic data and seismic modes associated with each option

10. One type of multicomponent seismic sensor that can be deployed on the seafloor to record multicomponent marine seismic data .........................................................16

11. Reflectivity equation for the SH-SH seismic mode............................................19

12. Analysis of $P$ and SV reflectivities requires that a polarity convention be assumed for

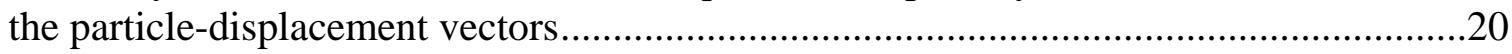

13. Each incident $\mathrm{P}$ and SV mode creates four scattered modes at an interface................20

14. Mathematical terms needed for P and SV reflectivity equations..............................21

15. Reflectivity equations for downgoing P-mode illumination....................................22

16. Reflectivity equations for downgoing SV-mode illumination................................22

17. Side-by-side comparison of 3-C and 9-C reflectivity equations...............................23 
18. Simplified formulation for P-wave reflectivity that can be used when two elastic media at an interface have "similar" petrophysical properties

19. Simplified formulation for SV reflectivity that can be used when two elastic media at an interface have "similar" petrophysical properties .25

20. Map view of particle-displacement wavefield propagating away from a horizontal-

displacement vector source

21. Map view of SH and SV illumination patterns for orthogonal horizontal-displacement sources....... .29

22. Section view of P-SV radiation pattern 30

23. Map view of P-SV illumination pattern.

24. Side-by-side comparison of 3-C and 9-C S-wave illumination patterns

25. Distinctions between 3-C SV-mode polarization and 9-C SV-mode polarization .......33

26. Distinction between 9-C CMP image points and 3-C CCP image points 37

27. Distinction between 3-C CCP image coordinates and 9-C CMP image point coordinates 39

28. Distinction between a 9-C SV-P CCP raypath and a 3-C P-SV CCP raypath.

29. Traveltimes for positive offsets are the same as traveltimes for negative offsets in 9-C CMP imaging because the lengths of the travel paths in Facies 1 and 2 are the same for both offset options.

30. Traveltimes for positive offsets are not the same as traveltimes for negative offsets in 3-C CCP imaging because the lengths of the P and SV raypaths in Facies 1 and 2 change when the offset direction changes.

31. Comparison of 9-C CMP image trace and 3-C CCP image trace.

32. Single vertical image trace in one stacking bin of CCP image space must be constructed by summing data from different time windows of all CCP traces that traverse the bin.

33. Simple, straight-raypath model showing that the velocity ratio $\mathrm{Vp} / \mathrm{Vs}$ in the propagation medium controls the position of a 3-C P-SV image coordinate in seismic image space.

34. 9-C SV-SV and SH-SH super gathers shown in field-record format .51 
35. 9-C SV-SV and SH-SH trace gathers after processing to emphasize primary reflections between 2 and $2.5 \mathrm{~s}$

36. Wave mode velocities calculated for transverse isotropic media showing that 9-C SV and SH velocities differ in a flat-layered Earth

37. Example 1 of polarities of 3-C P-SV data measured in azimuth directions that differ by $180^{\circ}$ 56

38. Example 2 of polarities of 3-C P-SV data measured in azimuth directions that differ

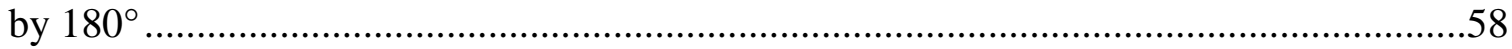

39. 2-D field record showing polarity behavior of the inline-horizontal component of PSV data. 60 


\section{INTRODUCTION}

Several concepts involved in generating, acquiring, and processing multicomponent seismic data are essential for understanding distinctions between 9component (9-C) and 3-component (3-C) data. The basic principle that has to be emphasized is that the physics of any multicomponent seismic technology cannot be understood until the data are viewed in terms of the displacement vector associated with each mode of the seismic wavefield that is being considered. This report therefore begins with a discussion of seismic vector-wavefield behavior to set the stage for all subsequent discussions.

There are three arguments that can be used to explain why each S-wave mode of 9-C and 3-C seismic data carries a different amount and a different type of rock/fluid information. These arguments were developed by scientists subcontracted to this study at the Bureau of Economic Geology (Bureau). One argument is designed to appeal to people who have limited interest in mathematics. The second approach is structured for people who have an appreciation of the mathematics of wavefield reflectivity. The third option is to illustrate the fundamental differences in the S-wave radiation patterns and S-wave target illuminations associated with 9-C and 3-C seismic sources. 


\section{EXECUTIVE SUMMARY}

This investigation summarizes the basic physics of nine-component (9-C) and three-component (3-C) shear-wave (S-wave) data and illustrates selected physical concepts of 9-C and 3-C S-waves with real data examples. There are fundamental differences in the P-SV S-wave mode provided by 3-C seismic data and the SH-SH and SV-SV S-wave modes available with 9-C data. Key distinctions among these S-wave modes are explained by describing differences in the sources that generate the modes, illustrating how the downgoing wavefields of the modes result in different illuminations of a target, showing differences in the reflectivity behaviors of the modes, and stressing how different source-receiver geometries and different data-processing strategies are required for 9-C data and for 3-C data. A principle that is stressed and illustrated repeatedly is that each mode of a multicomponent seismic wavefield may sense a different Earth fabric along its propagation path because the particle-displacement vector of each mode is oriented in a different direction. We conclude that because each wave mode has the potential of sensing an Earth fabric that its companion modes cannot, that optimal seismic evaluation of hydrocarbon prospects can occur only when 9-C seismic

data are acquired. 9-C seismic data provide all possible wave modes and all possible fabric-sensing options. 3-C seismic data provide only two fabric-sensing options: the P-P mode and the P-SV mode. 


\section{BASIC CONCEPTS THAT DISTINGUISH 9-C AND 3-C S-WAVE DATA}

The nonmathematical approach used to distinguish 9-C and 3-C S-wave data will be considered first. The logic of this argument emphasizes how differently Earth fabric can be sensed when a small rock volume embedded in a layered, spatially variant Earth is deformed in a different direction by the orthogonal displacement vectors associated with various seismic wave modes. Estimation of Earth fabric obtained from individual seismic wave modes can differ, and yet each estimate can be correct, because each mode deforms the test volume of rock in a different direction. These deformations sense different Earth resistance in directions parallel to, and normal to, various symmetry planes in real-Earth media. The logic of this nonmathematical model appeals in particular to people who are interested in only the geologic and petrophysical information that multicomponent seismic data may provide.

The second approach used to distinguish 9-C and 3-C wavefield behavior focuses on the mathematics of the reflectivity equation associated with each mode of the fullelastic seismic wavefield. The mathematical structure of the reflectivity equation associated with each seismic wave mode describes why and how petrophysical properties of the propagation medium affect different wave modes in different ways. The logic of this model is appreciated by geophysicists, engineers, and others who are comfortable with mathematics.

The third and last argument used to emphasize differences in 9-C and 3-C seismic data focuses on the S-wave illumination patterns produced by 9-C and 3-C seismic sources. The physics of the S-wave radiation associated with these sources is explained 
graphically, not numerically, to again have greater appeal to that large community of multicomponent seismic users who prefer not to be burdened by mathematical analyses.

All of these concepts led to the development of a new seismic interpretation science called elastic-wavefield seismic stratigraphy, which will be briefly described. The fundamental principle of elastic-wavefield seismic stratigraphy is that any mode of the elastic wavefield may provide unique rock, fluid, or sequence information across some stratigraphic intervals that cannot be obtained with the other wave modes of the elastic wavefield.

\section{Concept 1: Vector-Based Technology}

A special thought process based on vector concepts has to be used when developing and applying multicomponent seismic technology, regardless of whether the effort involves 9-C data or 3-C data. Previous seismic technology has been scalar based. For scalar data, it is not necessary to know the direction that each seismic wave mode moves the Earth. In multicomponent seismic technology, it is mandatory to know the direction of Earth displacement (vector-based thinking) when any step is taken to create, process, or interpret multicomponent data.

If the objective is to conduct a multicomponent seismic survey that will produce all possible wave modes, then each source station must be occupied by sources that generate three orthogonal source-displacement vectors. These three vectors must then propagate through the Earth as three independent illuminating wavefields. Such sources are called vector sources (Fig. 1). Full-vector source illumination requires that one illuminating wavefield (designated as wavefront 1 ) has a displacement vector oriented 
normal to its wavefront, and that two illuminating wavefields (designated as wavefronts 2 and 3) have orthogonal displacement vectors that are tangent to the respective wavefronts (Fig. 1). The displacement vector that is normal to wavefront 1 generates compressional (P-wave) data. The displacement vectors that are tangent to wavefronts 2 and 3 create shear (S-wave) data.

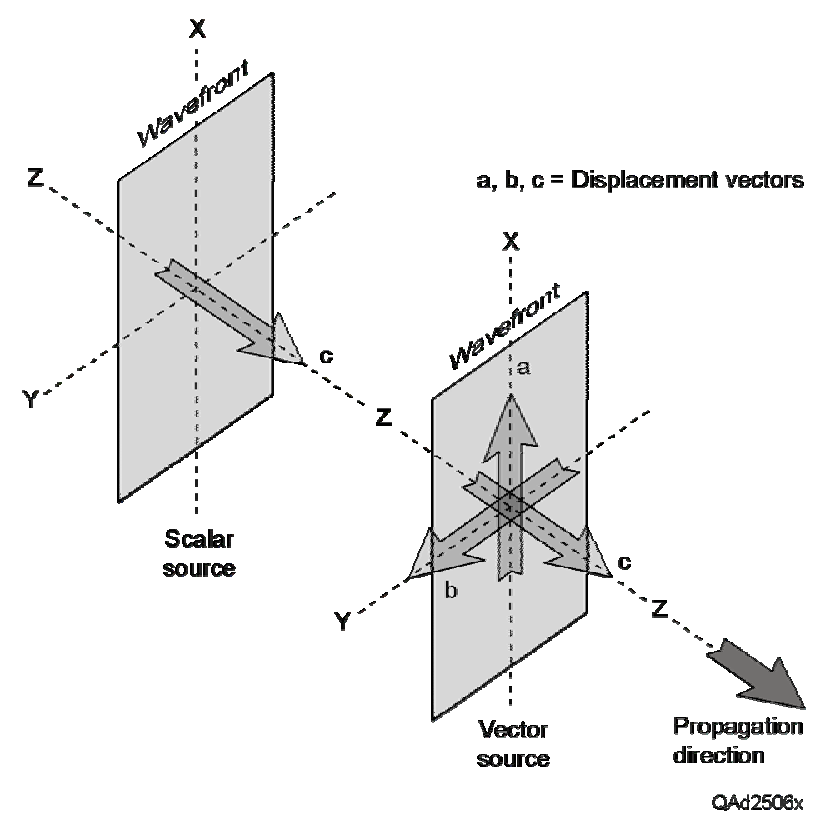

Figure 1. Distinction between vector and scalar seismic sources. A full-vector vector source should cause three orthogonal displacement vectors to propagate through the Earth. Two seismic properties are measured for a vector seismic source: the time-varying magnitude and the time-varying direction of the displacement of the Earth. A scalar source creates at least one displacement vector, but the seismic property that is measured is only the time-varying change of the magnitude of Earth movement, not the direction of that movement. 
An example of three vector-based vibrator sources positioned to create orthogonal source-displacement vectors is shown in Figure 2. In this example, a single vibrator is used to produce each of the three orthogonal source-displacement vectors illustrated for a vector source in Figure 1. A single-vibrator source is satisfactory in this instance because the data being acquired are 9-C vertical seismic profile (VSP) data, which do not require extreme, robust sources to produce good data quality. In large-scale 3-D seismic programs, arrays of vibrators may be needed to produce good-quality sourcedisplacement vectors at large offset distances. An example of 12 vibrators assembled for a 9C3D seismic survey is shown in Figure 3. In this instance, an array of four vertical vibrators produced the vertical-displacement source vector, an array of four horizontal vibrators produced the inline horizontal-displacement source vector, and a second array of four horizontal vibrators produced the crossline horizontal-displacement source vector. If the sources do not create these three orthogonal source-displacement vectors, some seismic wave modes of the full-elastic wavefield will not propagate into the Earth. The illuminating wavefields associated with the three orthogonal displacement vectors are produced and recorded in a time-sequence manner, with time delays of minutes to hours between generation of the vertical displacement vector, the inline horizontaldisplacement vector, and the crossline horizontal-displacement vector at each source station. 


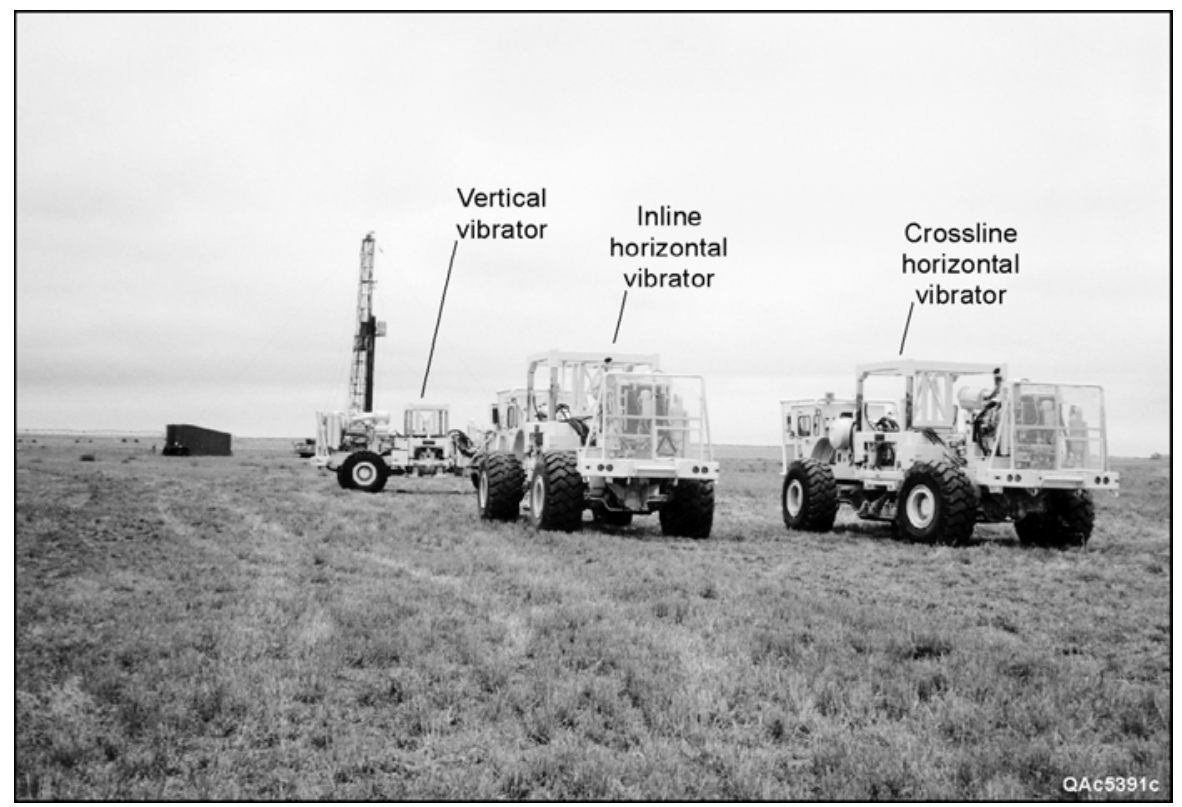

Figure 2. An example of three orthogonal seismic vector sources working to produce nine-component vertical seismic profile data. These three vibrators create the three source-displacement vectors illustrated for a vector seismic source in Figure 1 in a timesequence manner, not simultaneously.

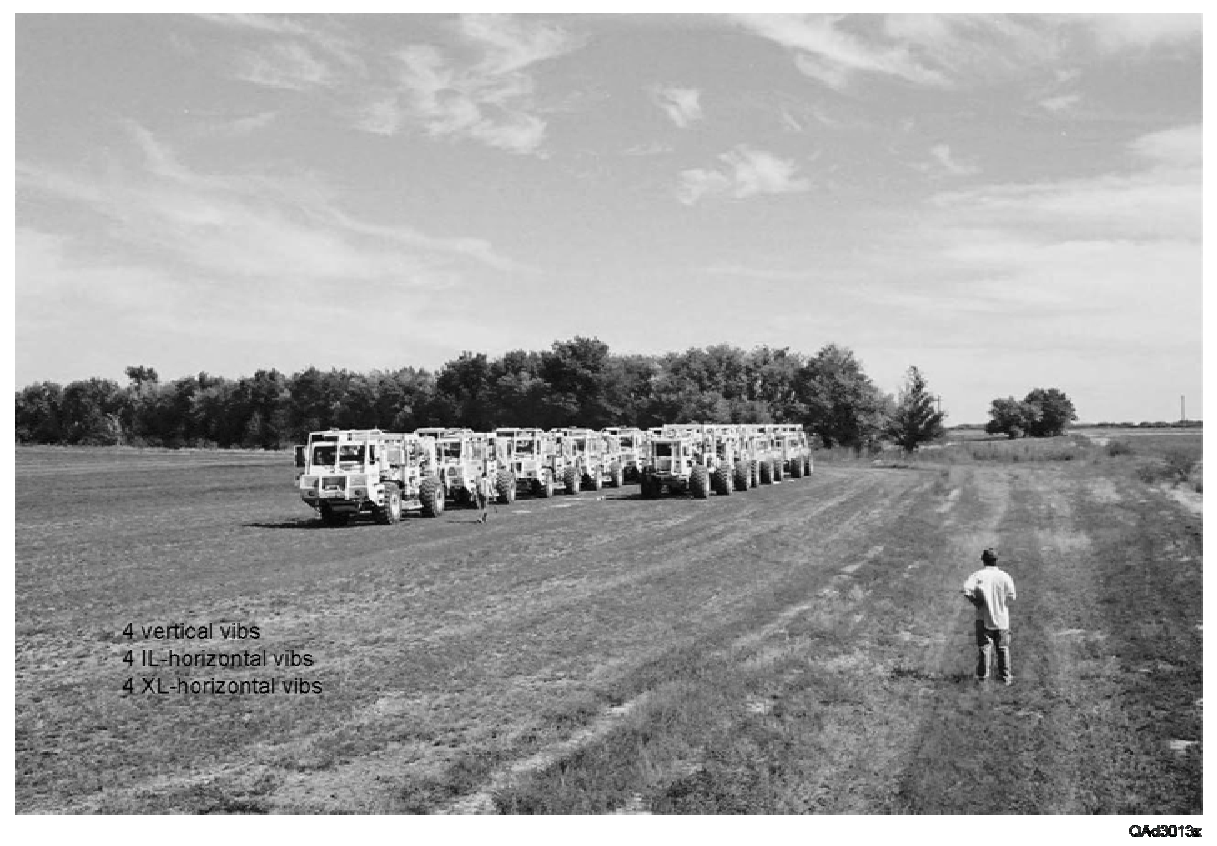

Figure 3. Twelve vector sources ready to deploy across a large 9C3D seismic survey. In this instance, four vibrators work as an array to produce a vertical source-displacement vector; four vibrators work in an array to produce an inline horizontal sourcedisplacement vector; and four vibrators work in an array to produce a crossline horizontal source-displacement vector. These three source-displacement vectors are produced in a time-sequence manner, not simultaneously, at each source station. 
Equally important, if there are not three orthogonal vector sensors at all receiver stations, then some wave modes produced by these three orthogonal source-displacement vectors will not be recorded. Three-component geophones are the oldest and most common type of vector sensor used to acquire multicomponent seismic data across onshore seismic prospects. A typical 3-C geophone is illustrated in Figure 4. This sensor package has one vertical moving-coil geophone element and two orthogonal and horizontal, moving-coil elements. A second vector-sensor technology based on solid-state accelerometers is now available and is being used in more and more multicomponent surveys. These sensors are called Micro-Electro-Mechanical System (MEMS) devices. The MEMS technology developed by Input/Output is illustrated in Figure 5. Sercel also offers MEMS 3-C vector sensors. Sercel’s concept for packaging MEMS vector-based sensors is illustrated in Figure 6.

(a)

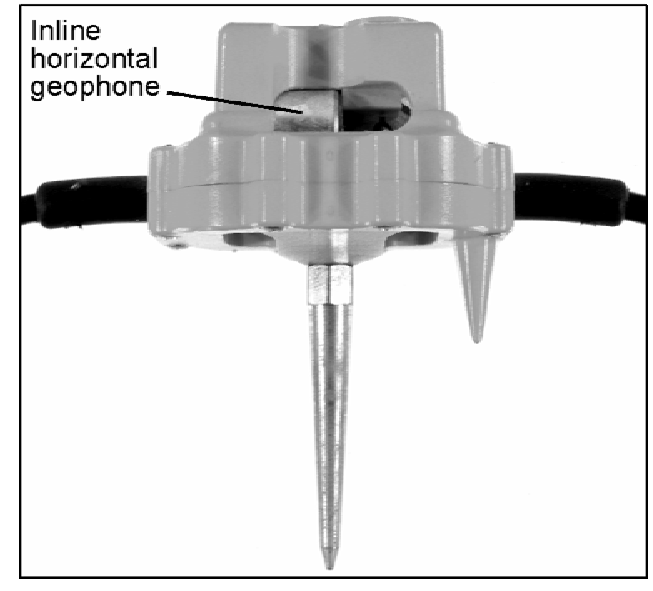

(b)

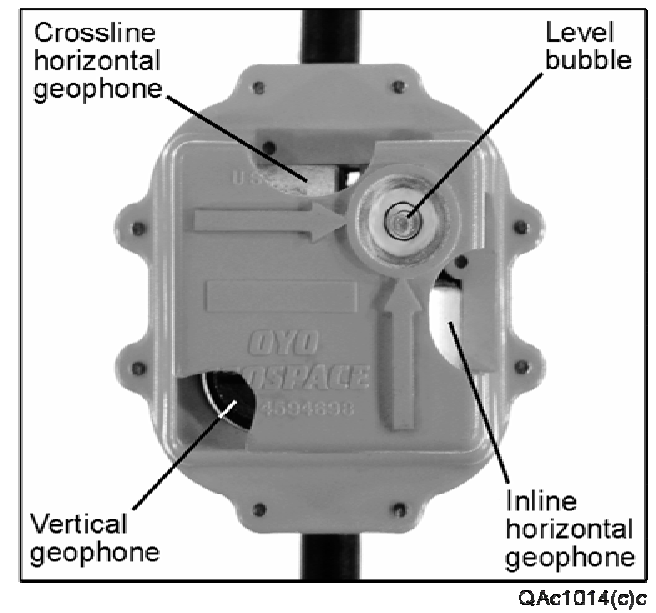

Figure 4. Standard three-component moving-coil geophone. 
Input/Output MEMS VectorSeis Sensor

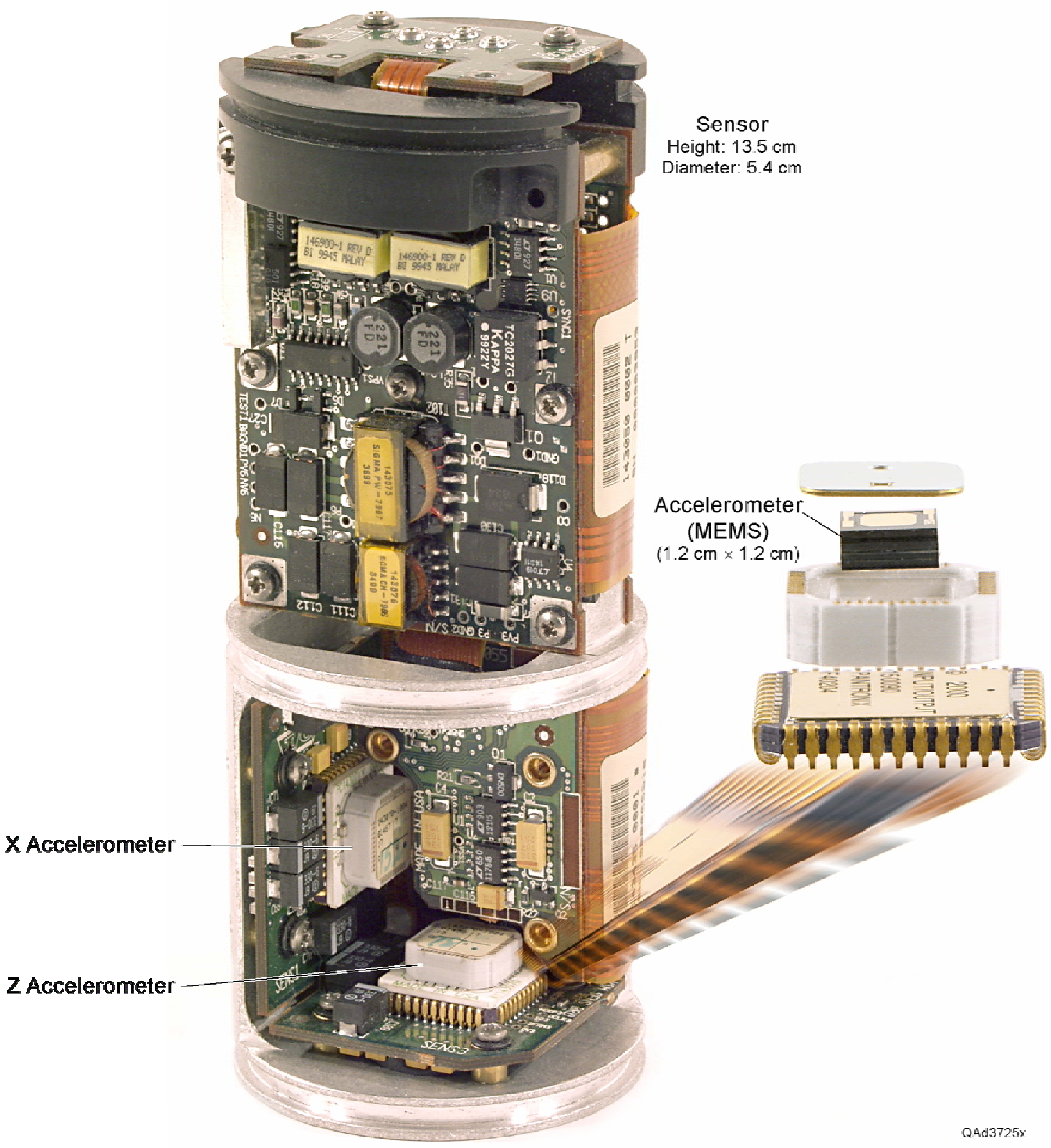

Figure 5. Micro-Electro-Mechanical System (MEMS) three-component seismic sensor available from Input/Output. The basic sensor element is a solid-state accelerometer. 


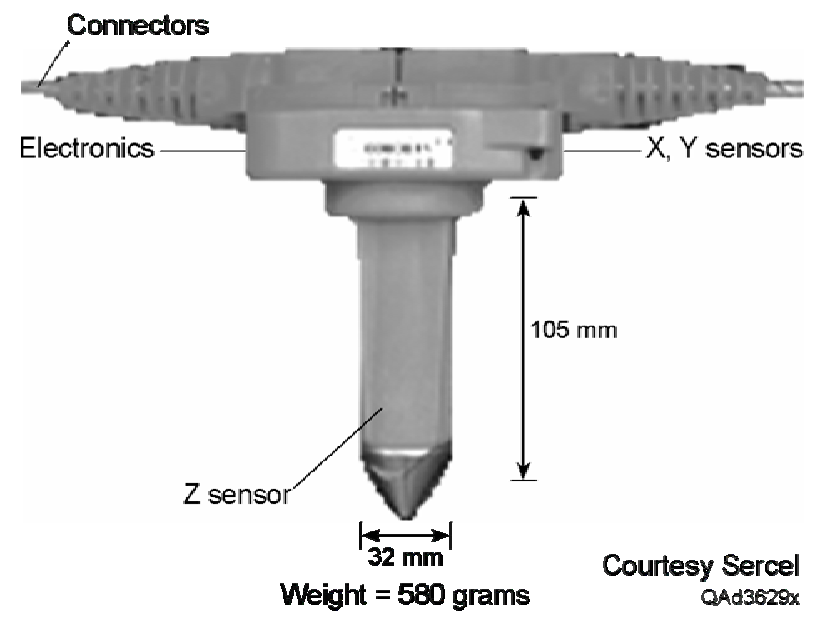

Figure 6. MEMS three-component sensor package available from Sercel.

If each source station is occupied by sources that create three orthogonal sourcedisplacement vectors (typically three different sources) and the wavefield produced by each source is then recorded by 3-C vector-based sensors, the result is a $9-C$ seismic vector wavefield. If the source station is occupied by a source that generates only one source-displacement vector (for example, sources such as a vertical vibrator or an explosive in a shothole in an onshore environment, or an air gun in a marine environment) and that single wavefield is then recorded by 3-C vector-based sensors, the result is a 3-C seismic wavefield. Distinctions between 9-C seismic wave modes and 3-C seismic wave modes will be emphasized throughout this report.

\section{Concept 2: Components That Make Multicomponent Seismic Data}

Three independent, vector-based, seismic wave modes propagate in a simple homogeneous Earth: a compressional mode, P, and two shear modes, SV and SH (Fig.7). These are the three modes we try to create with three orthogonal source-displacement vectors and then record with three orthogonal vector sensors. Each mode travels through 
the Earth at a different velocity, and each mode distorts the Earth in a different direction as it propagates. The propagation velocities of the SH and SV shear modes differ by only a few percent, but both shear velocities (Vs) are significantly less than the P-wave velocity (Vp). The velocity ratio $\mathrm{Vp} / \mathrm{Vs}$ can vary by an order of magnitude in Earth media, from a value of 15 in deep-water, unconsolidated, seafloor sediment to a value of 1.5 in a few dense, well-consolidated rocks. The orientations of the P, SV, and SH displacement vectors relative to the propagation direction of each mode are defined in Figure 7. A convenient way to distinguish between SH and SV shear modes is to imagine a vertical plane passing through a source station and a receiver station. SV vector displacement occurs in this vertical plane; $\mathrm{SH}$ vector displacement is normal to the plane (Fig. 8).

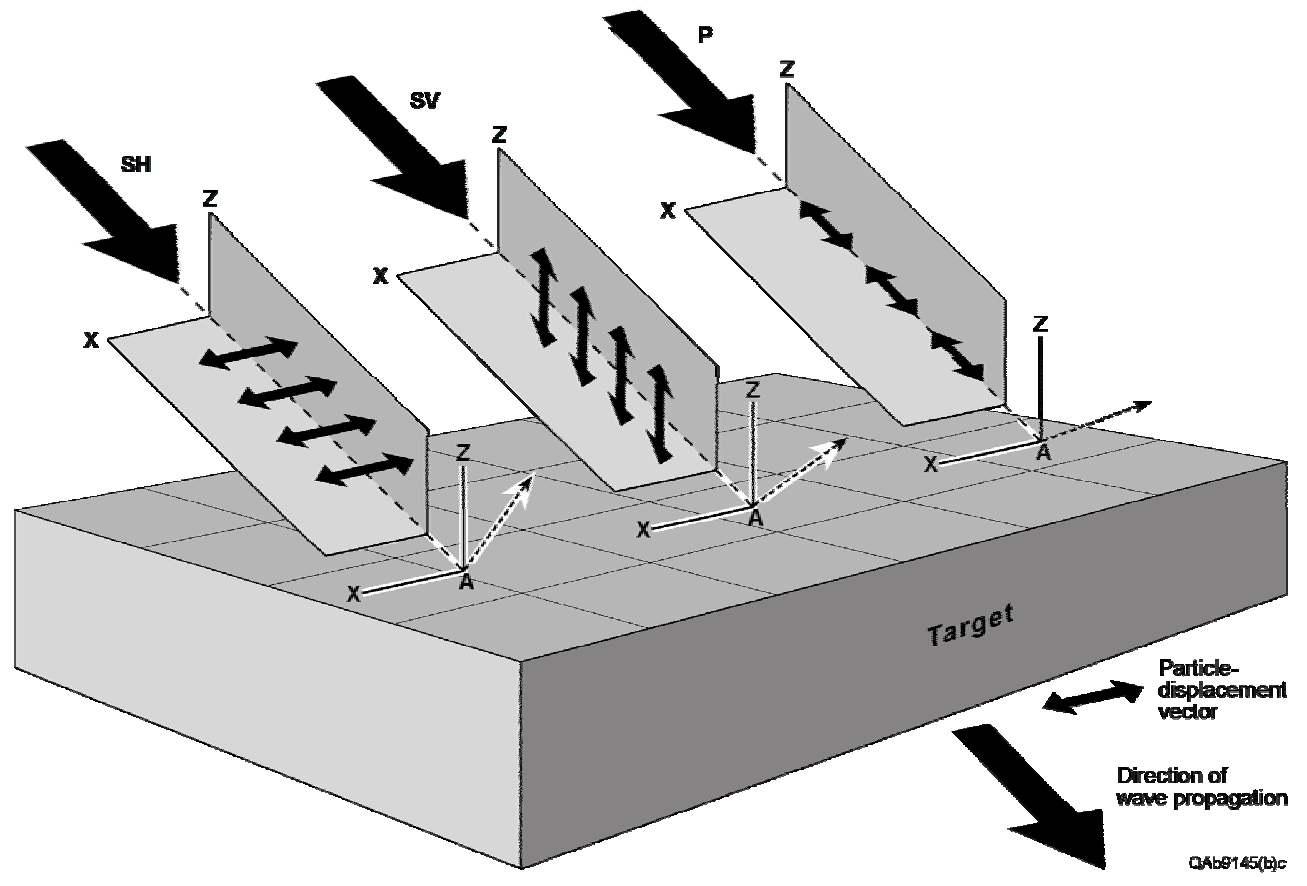

Figure 7. Full-elastic, multicomponent seismic wavefield propagating in a homogeneous Earth consisting of a compressional mode P and two shear modes, SV and SH. A key distinction among these modes is that each mode distorts the Earth in a different direction along its propagation path. The direction in which each mode distorts the Earth is indicated by the double-headed arrows. 


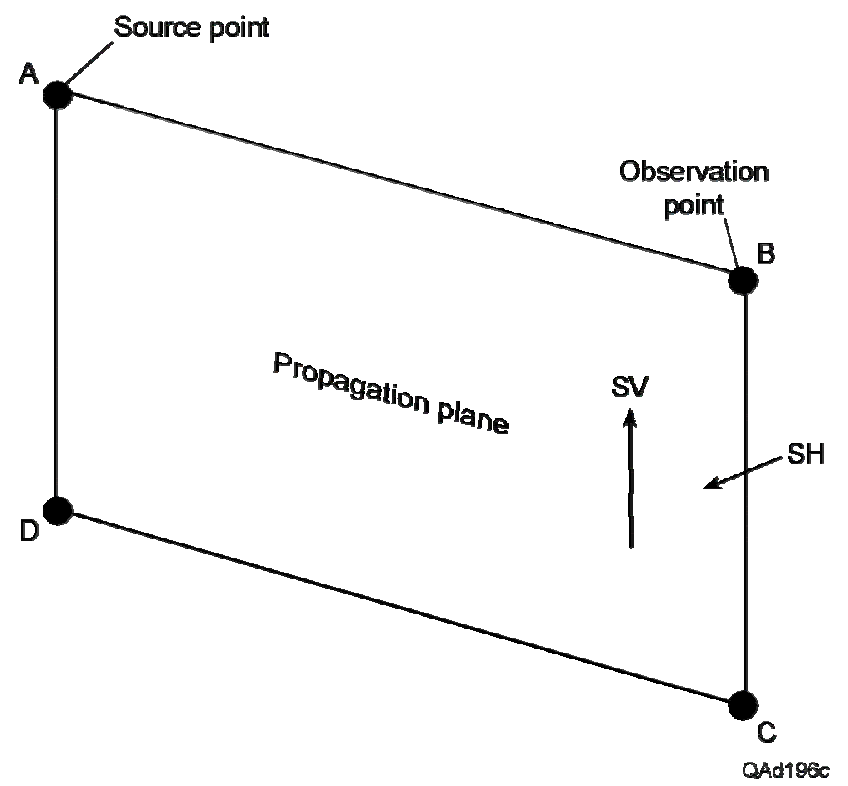

Figure 8. Distinction between SH and SV shear wave displacements. SV displacement occurs in the vertical plane that passes through the source station and the observation point; $\mathrm{SH}$ displacement is normal to this plane.

\section{Argument 1: Sensing the Earth Fabric}

We discuss now the first argument that can be used to distinguish how 9-C and 3C S-wave data sense petrophysical properties of the seismic propagation medium. This argument is based on the concept that the Earth’s fabric is a direction-dependent quantity.

In real Earth media, the physical character and elastic properties of the internal fabric of a small Earth volume depend on the direction in which the internal fabric of that volume is tested. Different elastic constants (fabric) are sensed when the Earth is distorted perpendicular to its bedding planes versus being displaced parallel to these planes, or when the Earth is displaced perpendicular to fractures versus parallel to fractures. For decades, the only seismic data used in oil and gas applications have been Pwave (scalar) data. The particle-displacement vector of a P-wave mode senses the Earth fabric in only one direction — the direction in which the P mode is propagating (Fig. 7). 
The advantage of multicomponent seismic data is that $\mathrm{P}, \mathrm{SH}$, and $\mathrm{SV}$ wave modes sense the Earth's fabric in three orthogonal directions (Fig. 7). Each wave mode thus carries unique Earth-fabric information, such as directional-dependent information about elastic constants, cementation quality, pore geometry, anisotropy axes, and lateral variations in rock and fluid types, as it leaves a target interval and travels to receiver stations. The technology challenges are to preserve this increased amount of geologic information when processing multicomponent seismic data and then to correctly interpret the geologic messages contained in the $\mathrm{P}, \mathrm{SH}$, and SV data volumes that are created.

\section{Terminology}

A new vocabulary is required to discuss multicomponent seismic technology. As previously stated, if three orthogonal source-displacement vectors are created at a source station (Figs. 2 and 3) and three orthogonal vector sensors record the distinct wavefields associated with each of these source displacements (Figs. 4 through 6), the result is ninecomponent data. Nine-component seismic data contain all possible wave modes. In this discussion, these wave modes will be designated as P-P, SH-SH, SV-SV, P-SV, and SVP. In this nomenclature, the term preceding the hyphen defines the downgoing wavefield, and the term following the hyphen specifies the upgoing wavefield. Three-component (3C) data are generated when three orthogonal vector sensors occupy the receiver stations but only a P-wave (1-C, or single displacement) source is used to generate the illuminating wavefield. Only two wave modes are provided by 3-C data: the P-P mode and the P-SV mode. 
A shear wave that propagates in an Earth that has vertical fractures, or that has a consistent tectonic orientation of the maximum horizontal stress vector, will segregate into two daughter modes called the fast-S mode and the slow-S mode. These daughter modes travel at different velocities, as their names imply, and they have orthogonal, not parallel, displacement vectors. The displacement vector of the fast-S mode is oriented parallel to the symmetry plane that is parallel to the vertical fractures (or parallel to the maximum horizontal stress if a stress condition is used to describe the propagation medium). The displacement vector of the slow-S mode is oriented normal to this symmetry plane. This wave physics is mentioned here only to complete this discussion of “terminology.” Examples of fast-S and slow-S data will not be included in this report.

The various options for acquiring multicomponent seismic data and the specific wave modes that are associated with each acquisition option are tabulated in Figure 9. Note how many S-wave modes are involved in multicomponent seismic data, particularly in fractured Earth media where S-wave splitting occurs. One terminology error encountered in multicomponent seismic applications is that people sometimes use the term "shear wave” and do not specify which particular shear mode is being considered. Each shear mode listed in Figure 9 is unique and commonly provides geologic information not available in its companion shear modes. Accurate terminology requires that we define the specific shear mode(s) we are dealing with in any multicomponent seismic operation. 


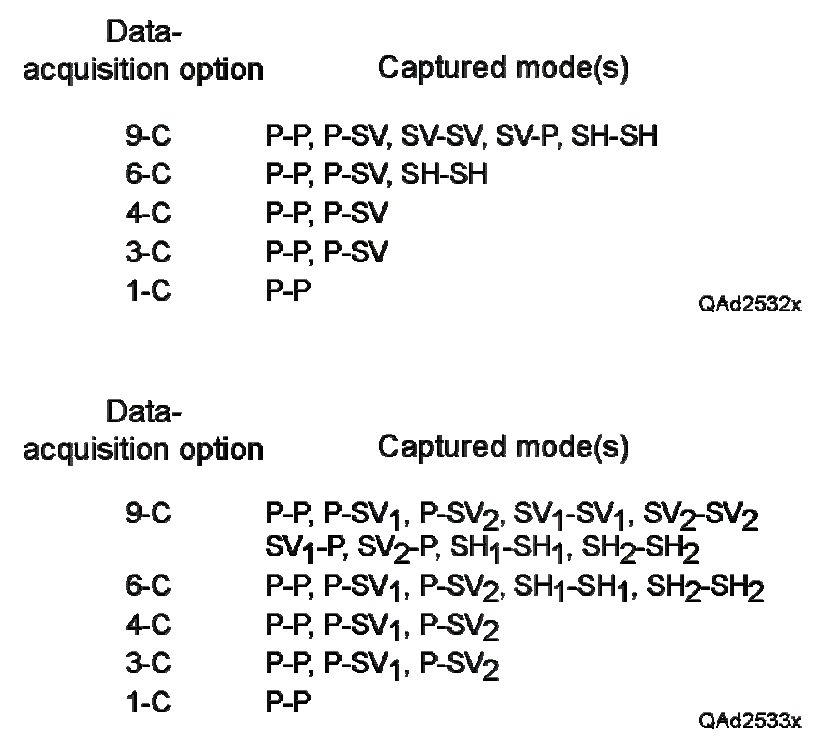

Figure 9. Options for acquiring multicomponent seismic data and seismic modes associated with each option. The top list applies to an isotropic Earth. The bottom list applies to an anisotropic medium in which S-wave splitting occurs. Subscript 1 defines a fast-S mode; subscript 2 indicates a slow-S mode.

In fact, our use of correct terminology matured during this investigation. For example, because the terms "SV," "SH," and "C-wave” were used in the title of the proposal that was submitted to DOE, those terms are used in the title of this report. We would now replace those terms, respectively, with the more accurate nomenclature "SVSV," “SH-SH,” and "P-SV."

\section{Marine Environments}

Shear waves cannot propagate in fluids, or in any media in which the shear modulus, $\mu$, has a value of zero. For multicomponent seismic data to be acquired in marine environments, sources and receivers need to be on the seafloor where they are in contact with sediment that has a nonzero value of $\mu$. To date, no vector-based sources function efficiently on the seafloor. The only source option for marine seismic data 
acquisition is an air-gun array suspended or towed in the water column. Such airgun sources will produce only P-wave (scalar) seismic wavefields in their water medium. Because the illuminating wavefield in a marine environment is limited to the $\mathrm{P}$ (scalar) mode, the only scattered wavefields that can be recorded are the P-P mode and the P-SV mode.

Several types of multicomponent, vector-based sensors can be deployed on the seafloor. One popular option is illustrated in Figure 10. As shown in this illustration, marine seafloor sensors contain three orthogonal, vector-sensing geophones, as well as a scalar-sensing hydrophone. Marine multicomponent seismic data are called fourcomponent data because the three components of geophone data are combined with the pressure data (scalar data) provided by the hydrophone. The fourth data component, pressure, is important because water-column multiples can be better suppressed by combining the vertical geophone response and hydrophone response.

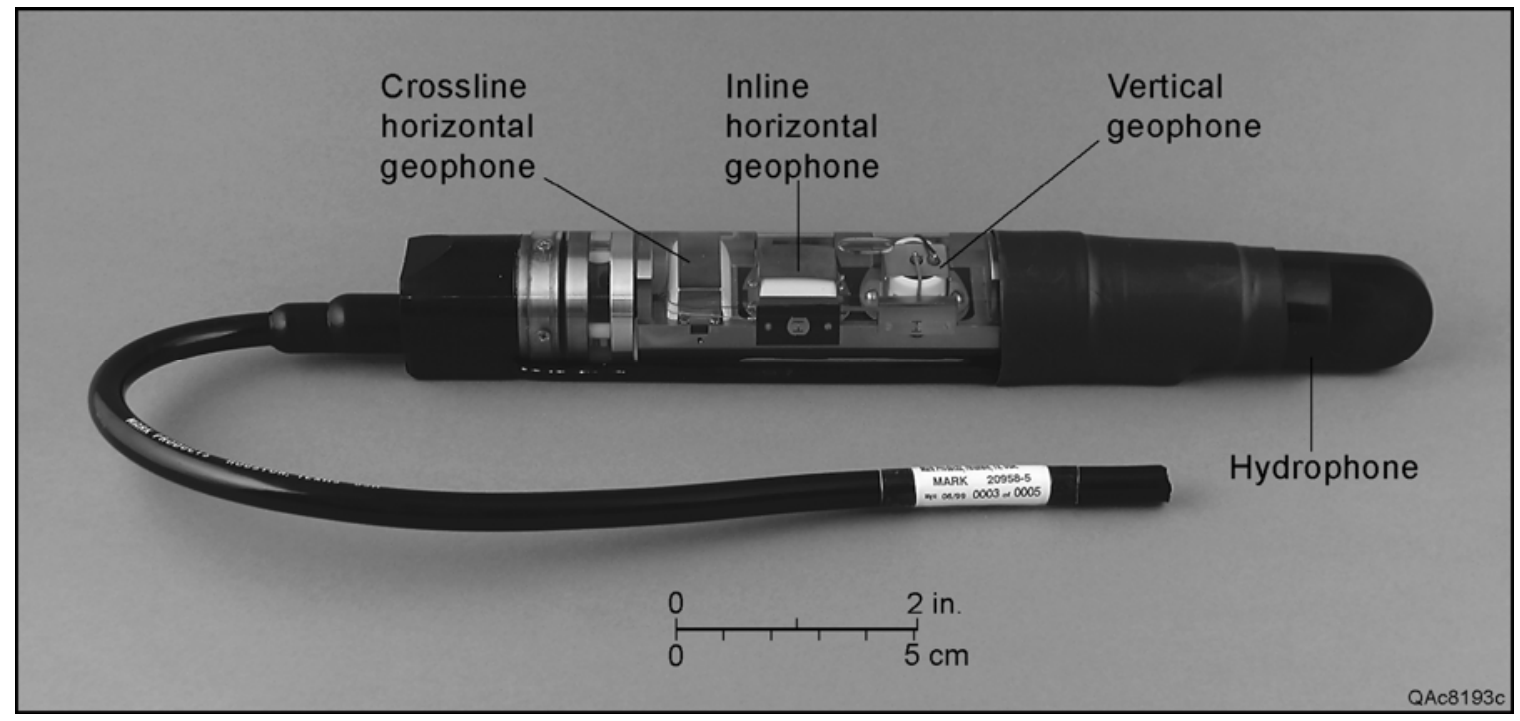

Figure 10. One type of multicomponent seismic sensor that can be deployed on the seafloor to record multicomponent marine seismic data. 


\section{Argument 2: Multicomponent Reflectivities}

We now move to the second argument that will be used to distinguish 9-C and 3C S-wave modes. This argument focuses on the reflectivity equations of multicomponent wave modes and involves some mathematics.

Each wave mode listed in Figure 9 has a unique reflectivity equation that relates the reflection amplitude and phase of that mode to elastic impedances of the Earth. These differing reflectivity equations are often the most compelling evidence to convince physicists, mathematicians, geophysicists, and other mathematically oriented investigators that elastic-wavefield seismic stratigraphy is built on a sound premise and that different Earth fabric is often sensed by each vector of the three orthogonal particledisplacement vectors involved in multicomponent seismic imaging.

Developing expressions for reflectivity equations of the various modes of a multicomponent seismic wavefield involves cumbersome and tedious algebra. The mathematics of reflectivity calculations is not particularly complex because it is essentially basic trigonometry and algebra. Yet many published analyses of reflectivity equations contain errors because the equations are lengthy, contain many terms, involve numerous petrophysical parameters, and provide multiple opportunities for making simple blunders, such as writing cosine when sine should be used, forgetting to include a parameter in an expression, inadvertently altering the algebraic sign of a term, or writing an incorrect subscript on parameters. Some of these published errors have persisted in the literature for years.

Because these types of errors are easy to make when reflectivity equations are calculated, most researchers copy the equations from a source that has proven over time 
to be error free. That approach will be followed in this discussion, using the widely accepted reflectivity equations published by Aki and Richards (1980).

A distinct reflectivity equation is needed for each of the wave modes listed in Figure 9. The simplest reflectivity equation is the one associated with the SH-SH mode, which is defined in Figure 11. Also shown in the figure is an illustration of the singleinterface Earth model that will be used in the derivation of all reflectivity equations. This model and the SH-SH reflectivity equation incorporate the notation for petrophysical properties used by Aki and Richards (1980). In Aki and Richard's nomenclature, alpha and beta represent P-wave and S-wave velocities, respectively. The terms Vp and Vs are used for these quantities in all other parts of this report. Additional petrophysical parameters are bulk density (rho), P-wave angle (i), S-wave angle (j), and horizontal slowness (p). Horizontal slowness is defined as

$$
\mathrm{p}=\sin (\mathrm{i}) / \mathrm{Vp}=\sin (\mathrm{j}) / \mathrm{Vs}
$$

Snell's law requires the horizontal slowness of every reflected and transmitted mode to be identical to the horizontal slowness of the incident wave that caused the reflection and transmission. Indices 1 and 2 attached to parameters refer, respectively, to the layer above the interface and to the layer below the interface. In Figure 11 and subsequent figures, subscripts $\mathrm{R}$ and $\mathrm{T}$ refer, respectively, to reflected and transmitted modes. The notation for these scattered SH modes is the same as the nomenclature used in Figure 9, except the hyphen is omitted. 


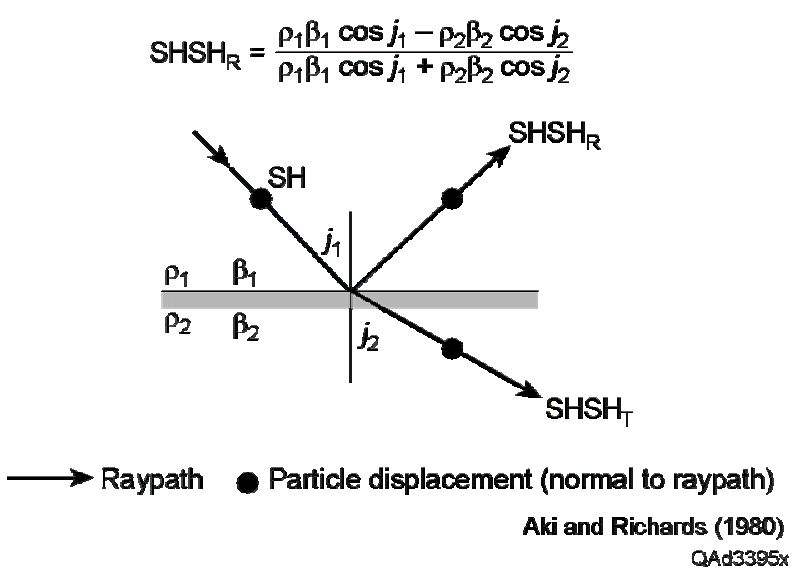

Figure 11. Reflectivity equation for the SH-SH seismic mode.

The development of reflectivity equations associated with seismic modes other than the SH-SH mode requires that a polarity convention be established for incident and transmitted P and SV particle-displacement vectors at an interface. The particledisplacement polarities assumed by Aki and Richards for P and SV modes are defined in Figure 12. If the particle-displacement vector of an incident, reflected, or transmitted P or SV mode points in the direction indicated for that mode in this illustration, the displacement vector has a positive algebraic sign. If the particle-displacement vector for a particular mode points in the opposite direction indicated by this model, that displacement vector has a negative algebraic sign. 


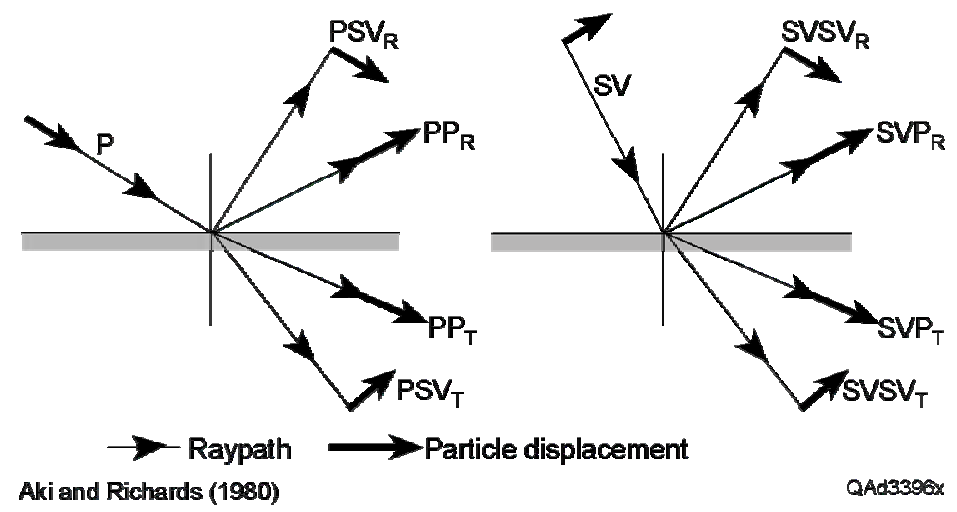

Figure 12. Analysis of $\mathrm{P}$ and SV reflectivities requires that a polarity (algebraic sign) convention be assumed for the particle-displacement vectors. The formulations for $\mathrm{P}$ and SV reflectivities that follow are based on the polarity convention shown here.

The Aki and Richards formulation of reflectivity equations allows both downgoingand upgoing-P and SV modes to be incident on the interface between two elastic layers. For each incident mode, four scattered wave modes are generated: upgoing $\mathrm{P}$, downgoing P, upgoing SV, and downgoing SV (Fig. 13). Relationships between the directions of P and SV wavefield propagation and the orientations of P and SV particle-displacement vectors that have positive algebraic signs are defined in this illustration.

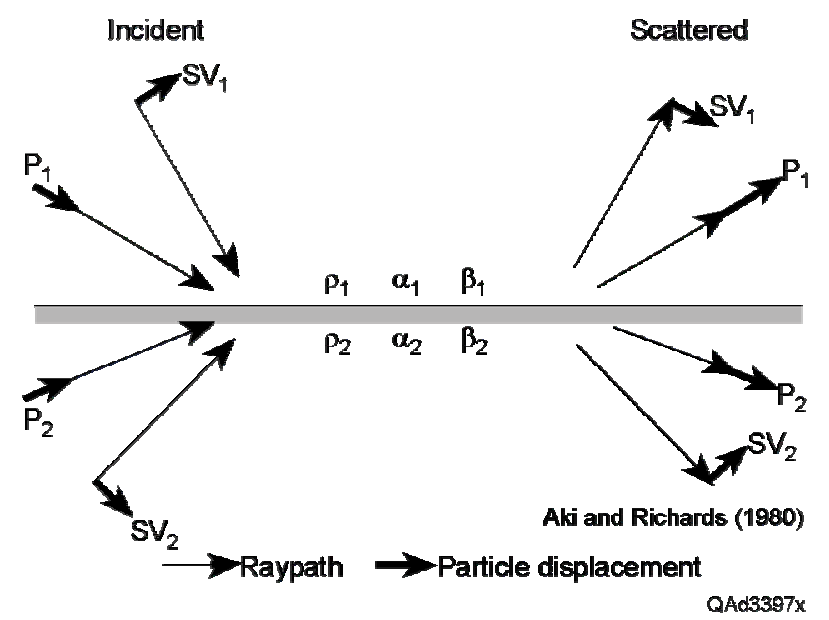

Figure 13. Each incident P and SV mode (left) creates four scattered modes (right) at an interface. 
By allowing four scattered modes for each of the four incident modes, Aki and Richards (1980) developed 16 equations to describe the total reflection/transmission physics of $\mathrm{P}$ and SV wavefields at an interface. Only 4 of these 16 equations are of interest in this discussion - the two reflectivity equations associated with a downgoing-P mode and the two reflectivity equations resulting from a downgoing-SV mode. To shorten the mathematical description of the reflectivity equations, Aki and Richards introduced the nine terms listed in Figure 14. With these terms being used, the reflectivity equations associated with a downgoing-P-mode illumination wavefield are then defined in Figure 15, and the two reflectivity equations produced by a downgoing-SV-mode illumination wavefield are given in Figure 16.

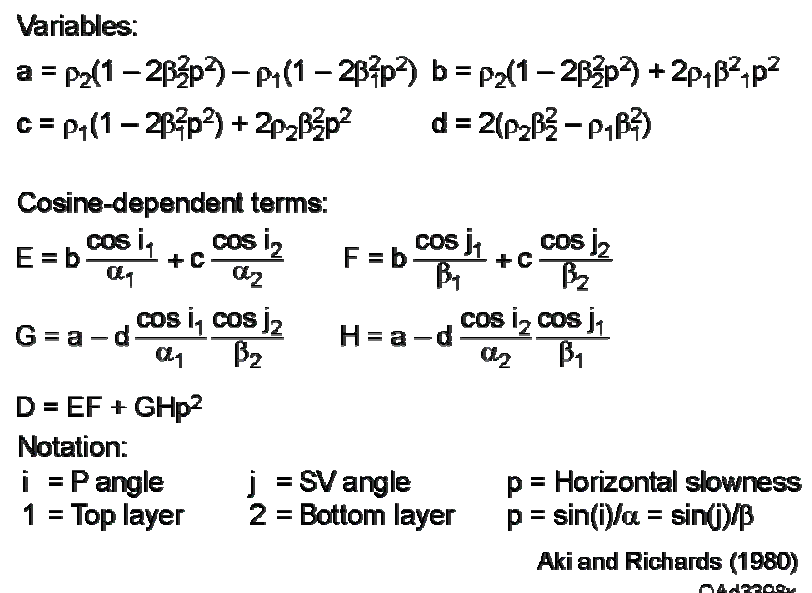

Figure 14. Mathematical terms needed for P and SV reflectivity equations. 


$$
\begin{aligned}
& P P_{R}=\left[\left(b \frac{\cos i_{1}}{\alpha_{1}}-c \frac{\cos i_{2}}{\alpha_{2}}\right) F-\left(a+d \frac{\cos i_{1}}{\alpha_{1}} \frac{\cos j_{2}}{\beta_{2}}\right) H p^{2}\right] / D \\
& \mathrm{PSV}_{\mathrm{R}}=-2 \frac{\cos \mathrm{i}_{1}}{\alpha_{1}}\left(\mathrm{ab}+\operatorname{cd} \frac{\cos \mathrm{i}_{2}}{\alpha_{2}} \frac{\cos \mathrm{j}_{2}}{\beta_{2}}\right) \mathrm{p \alpha _{1 }} /\left(\beta_{1} \mathrm{D}\right) \\
& \mathbf{i}=\mathbf{P} \text { angle } \quad \mathbf{j}=\text { SV angle } \quad \mathrm{p}=\text { Horizontal slowness } \\
& 1=\text { Top layer } \quad 2=\text { Bottom layer } \quad p=\sin (i) / \alpha=\sin (j) / \beta \\
& \begin{array}{r}
\text { Aki and Richards (1980) } \\
\text { QAd3399x }
\end{array}
\end{aligned}
$$

Figure 15. Reflectivity equations for downgoing P-mode illumination. These two reflectivity equations are the ones of interest in 3-C and 4-C seismic imaging. Terms $\mathrm{a}, \mathrm{b}$, c, d, D, F, and $\mathrm{H}$ are defined in Figure 14. Horizontal slowness is defined by Equation 1 in the text. Note how complicated these expressions are compared with the reflectivity equation for the SH mode in Figure 11.

$$
\begin{aligned}
& S V P_{R}=2 \frac{\cos j_{2}}{\beta_{2}}\left(a c+b d \frac{\cos i_{1}}{\alpha_{1}} \frac{\cos j_{1}}{\beta_{1}}\right) p \beta_{2} /\left(\alpha_{2} D\right) \\
& \text { SVSV }_{R}=\left[\left(b \frac{\cos j_{1}}{\beta_{1}}-c \frac{\cos j_{2}}{\beta_{2}}\right) E+\left(a+d \frac{\cos i_{1}}{\alpha_{1}} \frac{\cos j_{2}}{\beta_{2}}\right) H p^{2}\right] / D \\
& \mathrm{i}=\mathrm{P} \text { angle } \quad \mathrm{j}=\mathrm{SV} \text { angle } \quad \mathrm{p}=\text { Horizontal slowness } \\
& 1=\text { Top layer } \quad 2=\text { Bottom layer } \quad \mathbf{p}=\sin (\mathrm{i}) / \alpha=\sin (\mathrm{j}) / \beta \\
& \text { Aki and Richards (1980) }
\end{aligned}
$$

Figure 16. Reflectivity equations for downgoing SV-mode illumination. Terms a, b, c, d, $\mathrm{D}, \mathrm{E}$, and $\mathrm{H}$ are defined in Figure 14. Horizontal slowness is defined by Equation 1 in the text. Compare the complexity of these expressions with the simpler expression for the reflectivity equation of the SH mode in Figure 11.

All wave modes listed in Figures 15 and 16 have a subscript R because we are interested in only reflected wavefields in this discussion. The notation used to identify these reflected modes is identical to the nomenclature in Figure 13 and in Figure 9 (with the hyphen omitted). The reflectivity equations in Figure 15 are of particular interest because they describe the P-P and P-SV modes involved in 3-C and 4-C seismic technology (Fig. 9). 
Any downgoing-wave mode could be used to acquire 3-C seismic data, but in this report, 3-C and 4-C seismic technology will be restricted to data produced by only Pwave illumination. This definition of 3-C and 4-C seismic data is standard practice in the seismic industry. The reflectivity equations in Figures 11 and 16 thus apply to 9-C seismic technology, not 3-C seismic technology, because they are produced, respectively, by SH-mode and SV-mode illumination, not by P-mode illumination. To simplify the comparison of the reflectivity physics associated with each 3-C and 9-C seismic mode, the foregoing equations are positioned in a side-by-side format in Figure 17. The left column describes 3-C (or 4-C) reflectivity. The right column describes 9-C reflectivity.

\begin{tabular}{|c|c|}
\hline 3-C technology & 9-C technology \\
\hline $\begin{array}{l}P P_{R}=\left[\left(b \frac{\cos i_{1}}{\alpha_{1}}-c \frac{\cos i_{2}}{\alpha_{2}}\right) F-\left(a+d \frac{\cos i_{1}}{\alpha_{1}} \frac{\cos j_{2}}{\beta_{2}}\right) H p^{2}\right] / D \\
P S V_{R}=-2 \frac{\cos i_{1}}{\alpha_{1}}\left(a b+c d \frac{\cos i_{2}}{\alpha_{2}} \frac{\cos j_{2}}{\beta_{2}}\right) P \alpha_{1} /\left(\beta_{1} D\right)\end{array}$ & $\begin{aligned} P P_{R} & =\left[\left(b \frac{\cos i_{1}}{\alpha_{1}}-c \frac{\cos i_{2}}{\alpha_{2}}\right) F-\left(a+d \frac{\cos i_{1}}{\alpha_{1}} \frac{\cos j_{2}}{\beta_{2}}\right) H p^{2}\right] / D \\
P S V_{R} & =-2 \frac{\cos i_{1}}{\alpha_{1}}\left(a b+c d \frac{\cos i_{2}}{\alpha_{2}} \frac{\cos j_{2}}{\beta_{2}}\right) p \alpha_{1} /\left(\beta_{1} D\right)\end{aligned}$ \\
\hline & $\begin{aligned} S V P_{R} & =2 \frac{\cos j_{2}}{\beta_{2}}\left(a c+b d \frac{\cos i_{1}}{\alpha_{1}} \frac{\cos j_{1}}{\beta_{1}}\right) p \beta_{2} /\left(\alpha_{2} D\right) \\
s V S V_{R} & =\left[\left(b \frac{\cos j_{1}}{\beta_{1}}-c \frac{\cos j_{2}}{\beta_{2}}\right) E+\left(a+d \frac{\cos i_{1}}{\alpha_{1}} \frac{\cos j_{2}}{\beta_{2}}\right) H p^{2}\right] / D\end{aligned}$ \\
\hline & $\mathrm{SHSH}_{\mathrm{R}}=\frac{\rho_{1} \beta_{1} \cos j_{1}-\rho_{2} \beta_{2} \cos j_{2}}{\rho_{1} \beta_{1} \cos j_{1}+\rho_{2} \beta_{2} \cos j_{2}}$ \\
\hline $\begin{array}{ll}\mathrm{j}=\text { SV angle } & \mathrm{p}=\text { Horizontal slowness } \\
2=\text { Bottom layer } & \mathrm{p}=\sin (\mathrm{i}) / \alpha=\sin (\mathrm{j}) / \beta\end{array}$ & Aki and Richards (1980) \\
\hline
\end{tabular}

Figure 17. Side-by-side comparison of 3-C and 9-C reflectivity equations.

Key principles illustrated by these equations can now be noted.

1. 3-C seismic data are a subset of 9-C seismic data (P-P and P-SV modes: top box of both columns). 
2. Only one S-wave mode (P-SV) is provided by 3-C data; 9-C data provide three S-wave modes (SV-SV and SH-SH, as well as P-SV).

3. The reflectivity equations for the three S-wave modes (P-SV, SV-SV, SH-SH) differ from each other. Each $S$ mode may thus result in a different image of the subsurface, even though all three images can be correct in terms of their reflectivity physics.

4. The SV shear mode and the P compressional mode are linked to each other, and energy is exchanged between these two modes during reflection.

5. The SH shear mode is not linked to either P or SV, and no energy exchange between $\mathrm{SH}$ and these modes occurs during reflection.

6. The only way to generate a reflected $\mathrm{SH}$ mode is to use an $\mathrm{SH}$ source for illumination. An SH mode is thus never available in 3-C or 4-C seismic data because the data are generated by a P source.

7. SH-SH reflectivity is simpler (mathematically) than SV-SV and P-SV reflectivities. This fact implies that $\mathrm{SH}$ shear-wave data should be easier to process and interpret than SV-SV and P-SV data.

8. Only one P-wave mode (P-P) is available with 3-C data; 9-C data provide two $\mathrm{P}$-wave modes (P-P and SV-P).

This analysis leads to the conclusion that differences in mathematical structure of the reflectivity equations for the various seismic wave modes cause these modes to react to changes in elastic constants in different ways. The result is that one mode sometimes images stratal surfaces and produces seismic sequences and facies that are different from those of the other modes. This fact is particularly important when assessing the relative value of 3-C and 9-C S-wave imaging. Because 9-C data allow three independent S-wave images to be made but 3-C data provide only one S-wave image, 9-C S-wave data should always provide more petrophysical, stratigraphic, sequence, and facies information than should 3-C data.

The complex reflectivity equations associated with illuminating P and SV modes can be simplified when the petrophysical properties of the two Earth layers at an interface 
are "similar." The definition of "similar" Earth parameters is arbitrary, but in most instances it is reasonable to assume that a variation of less than 20 percent in bulk density (rho) and in velocities Vp and Vs across a boundary satisfies the approximation of similarity between the two Earth layers at that boundary. In such instances, the lengthy, tedious mathematical descriptions of reflectivity equations for an illuminating P mode (Fig. 15) simplify to the expressions in Figure 18. The reflectivity equations for an illuminating SV mode reduce to the simpler expressions in Figure 19. These simplified equations are adequate for most multicomponent seismic modeling exercises and for most multicomponent seismic data analyses. They also allow density-contrast and velocity-contrast contributions to reflectivity to be compared more easily than do the equations in Figures 15 and 16.

$$
\begin{aligned}
& \mathrm{PP}_{\mathrm{R}}=\frac{1}{2}\left(1-4 \beta^{2} \mathrm{p}^{2}\right) \frac{\Delta p}{\rho}+\frac{1}{2 \cos ^{2} \frac{\Delta \alpha}{\alpha}}-4 \beta^{2} \mathrm{p}^{2} \frac{\Delta \beta}{\beta} \\
& P S V_{R}=\frac{-p \alpha}{2 \cos j}\left[\left(1-2 \beta^{2} p^{2}+2 \beta^{2} \frac{\cos i}{\alpha} \frac{\cos j}{\beta}\right) \frac{\Delta p}{\rho}\right. \\
& \left.-\left(4 \beta^{2} p^{2}-4 \beta^{2} \frac{\cos i}{\alpha} \frac{\cos j}{\beta}\right) \frac{\Delta \beta}{\beta}\right] \\
& \alpha, \beta, \rho=\text { Mean values } \quad \Delta \alpha, \Delta \beta, \Delta p=[(\text { Value } 2)-(\text { Nalue 1) }] \\
& i=P \text { angle } \\
& \mathrm{j}=\mathrm{SV} \text { angle } \\
& \text { p = Horizontal slowness } \\
& p=\sin (i) / \alpha=\sin (j) / \beta
\end{aligned}
$$

Figure 18. Simplified formulation for P-wave reflectivity that can be used when two elastic media at an interface have "similar" petrophysical properties. Horizontal slowness is defined by Equation 1 in the text.

$$
\begin{aligned}
& \operatorname{SVP}_{R}=\frac{\cos j}{\alpha} \frac{\beta}{\cos i} \mathrm{PSV}_{\mathrm{R}} \text {, } \\
& \operatorname{SVSV}_{R}=-\frac{1}{2}\left(1-4 \beta^{2} \mathrm{p}^{2}\right) \frac{\Delta p}{\rho}-\left(\frac{1}{2 \cos ^{2} j}-4 \beta^{2} \mathrm{p}^{2}\right) \frac{\Delta \beta}{\beta} \\
& \alpha, \beta, \rho=\text { Mean values } \quad \Delta \alpha, \Delta \beta, \Delta \rho=[\text { (Value } 2)-(\text { Value 1)] } \\
& \mathrm{i}=\mathrm{P} \text { angle } \\
& \mathrm{j}=\mathrm{SV} \text { angle } \\
& \mathrm{p}=\text { Horizontal slowness } \\
& \mathrm{p}=\sin (\mathrm{i}) / \alpha=\sin (\mathrm{j}) / \beta \\
& \text { Aki and Richards (1980) }
\end{aligned}
$$

Figure 19. Simplified formulation for SV reflectivity that can be used when two elastic media at an interface have "similar" petrophysical properties. Horizontal slowness is defined by Equation 1 in the text. 
Argument 3: Multicomponent Illumination

The preceding section discussed distinctions between the reflected S-wave modes involved in 9-C and 3-C seismic data acquisition. To further appreciate how 3-C and 9-C S-wave data differ, it is equally important to consider distinctions between the downgoing illumination patterns of 9-C and 3-C S-wave modes. The reflectivity equations developed in the previous section assume that the illuminating wave mode, whether it is a P, SV, or SH mode, is a plane wave. In discussing this final argument, we will consider S-wave radiation patterns generated by finite sources.

A map view of the particle-displacement wavefield produced by a horizontaldisplacement vector source is illustrated in Figure 20. It is assumed that the source introduces a horizontal displacement oriented from left to right over the finite Earth-tosource contact area, labeled S. This source-displacement vector converts into the particledisplacement vectors shown distributed over the image space. All particle-displacement vectors are drawn with equal length because the intent of this illustration is to show orientations of the vectors across the image space, not their relative magnitudes. The key point is that at every image coordinate encircling the source station, the particledisplacement vector is always oriented in the direction of the source-displacement vector. Bold arrows G1 through G4 indicate the positive orientation direction of a horizontal vector sensor at four locations around the source station. The particle-displacement vector at each sensor station is oriented in the direction of positive sensor response. The principle illustrated in Figure 8 will be used to define SV and SH shear modes produced by this vector source. If a vertical plane is constructed through source station S and 
sensor stations G2 and G4, the propagating particle-displacement vector is constrained to that plane. Thus sensors G2 and G4 measure SV shear motion (Fig. 8). If a vertical plane is constructed through the source station and sensor stations G1 and G3, the particledisplacement vector is normal to that plane. Sensors G1 and G3 thus measure SH shear motion (Fig. 8). If a vertical plane is constructed through the source station and arbitrary point $\mathrm{A}$, the particle-displacement vector has components parallel to, and normal to, the plane. A sensor at point A would thus record a mixture of SV and SH shear motion. This exercise demonstrates that a single horizontal-displacement source produces both SH and SV modes, and that these modes radiate away from the source station in asymmetrical patterns. The proportions of SH and SV energies that arrive at an image coordinate vary with azimuth from the source station to the image point.

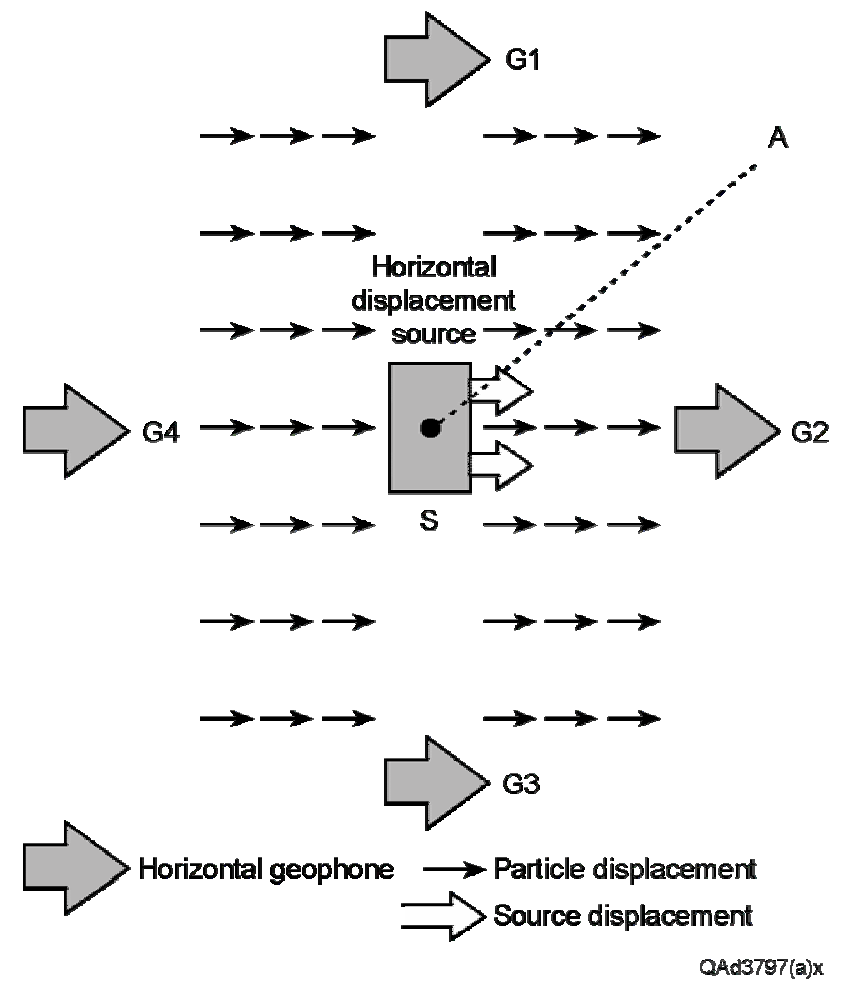

Figure 20. Map view of particle-displacement wavefield propagating away from a horizontal-displacement vector source. 
Numerous people have developed mathematical expressions that describe the geometrical shape of $\mathrm{P}, \mathrm{SV}$, and $\mathrm{SH}$ radiation patterns produced by seismic sources in an isotropic Earth. One of the respected references on this topic is White (1983). These published analyses show that in map view, SH and SV radiation patterns produced by a horizontal-displacement source have the appearance of that shown in Figure 21. Viewed from directly above the horizontal-displacement source, SV and SH modes propagate away from the source station as expanding circles. Because SV radiation from a horizontal-displacement source is more energetic than $\mathrm{SH}$ radiation, $\mathrm{SV}$ radiation circles are drawn larger than SH radiation circles. These circles indicate which parts of the image space each mode affects and the magnitude of the mode illumination that reaches each image coordinate. For example, a horizontal source-displacement vector oriented in the $\mathrm{Y}$ direction (left side of figure) causes $\mathrm{SV}$ modes to radiate in the $+\mathrm{Y}$ and $-\mathrm{Y}$ directions and $\mathrm{SH}$ modes to propagate in the $+\mathrm{X}$ and $-\mathrm{X}$ directions. A horizontal sourcedisplacement vector oriented in the $\mathrm{X}$ direction (right side of figure) causes SV modes to radiate in the $+\mathrm{X}$ and $-\mathrm{X}$ directions and $\mathrm{SH}$ modes to propagate in the $+\mathrm{Y}$ and $-\mathrm{Y}$ directions. If a line is drawn from the source station to intersect one of these radiation circles, the distance to the intersection point indicates the magnitude of that particular mode displacement in the azimuth direction of that line. The orientation of the particledisplacement vector remains constant across the image space, as indicated in Figure 20, but the magnitude of the SH and SV particle-displacement vectors vary with azimuth as shown, respectively, by the SH and SV radiation circles in Figure 21. 


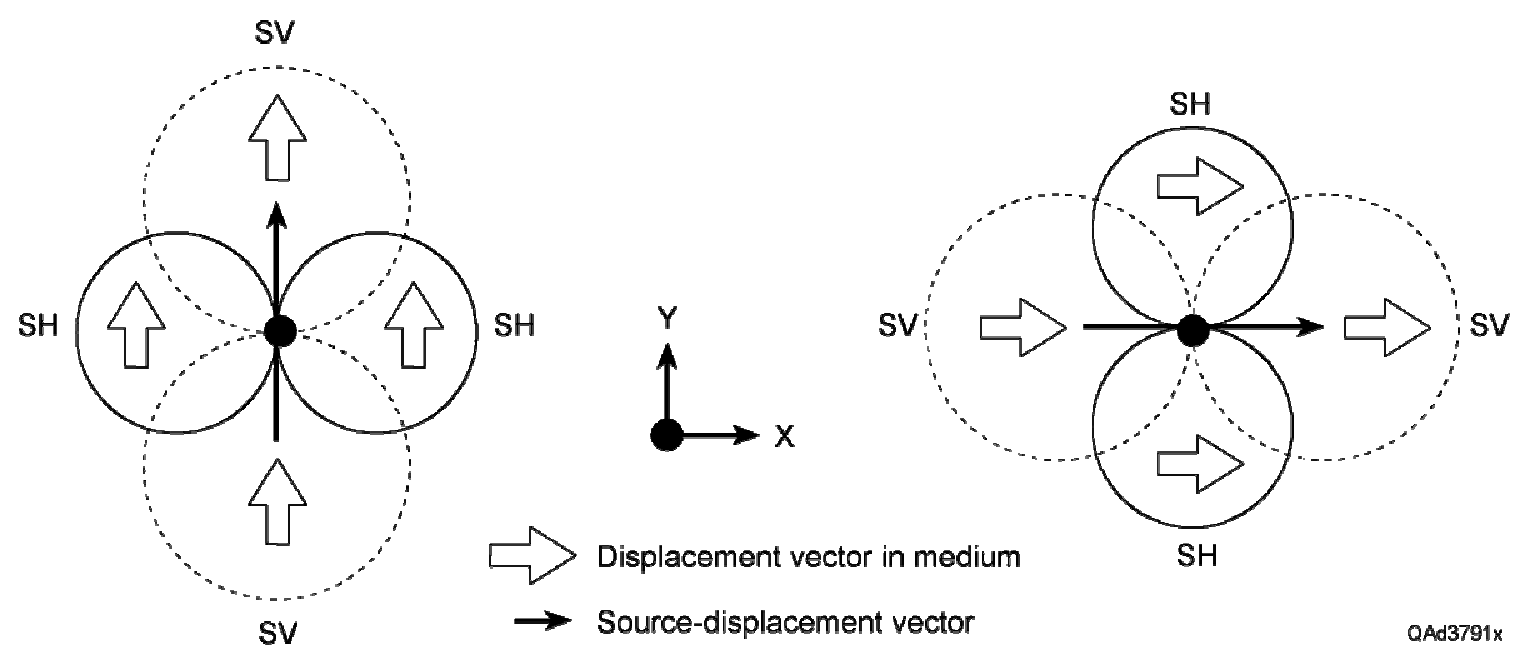

Figure 21. Map view of SH and SV illumination patterns for orthogonal (X and Y) horizontal-displacement sources.

The shear-wave radiation associated with P-to-SV mode conversion is much different from that produced by a horizontal-displacement source. Section and map views of P-SV radiation patterns are provided as Figures 22 and 23, respectively. The section view (Fig. 22) indicates an air gun operating in a water environment (a scalar source). The converted-SV radiation patterns in this diagram apply equally well to land-based operations where the energy source is a vertical vibrator or an explosive in a shot hole. In both 3-C (land) and 4-C (marine) data acquisition, the SV radiation pattern associated with the P-SV mode is produced in the subsurface at the P-to-SV conversion point, not at the surface-based source station, as is the case for a horizontal-displacement source (Figs. 20 and 21). The map view in Figure 23 shows the downgoing-P mode propagating away from the source station, with SV radiation patterns being produced at subsurface interfaces at every point along the $\mathrm{P}$ wavefront. The dotted patterns indicate the geometrical shape of the converted-SV radiation that is created at each subsurface P-to- 
SV conversion point. A key point to note is that the orientation of the SV particledisplacement vector is not in a fixed direction, as it is for a horizontal-displacement source (Fig. 20), but varies with azimuth direction. The vector orientations shown in this diagram are correct for an isotropic Earth where the total SV displacement is oriented in the radial direction in which the P wave is propagating. In an anisotropic Earth, the SV particle-displacement vector has both radial and transverse components.

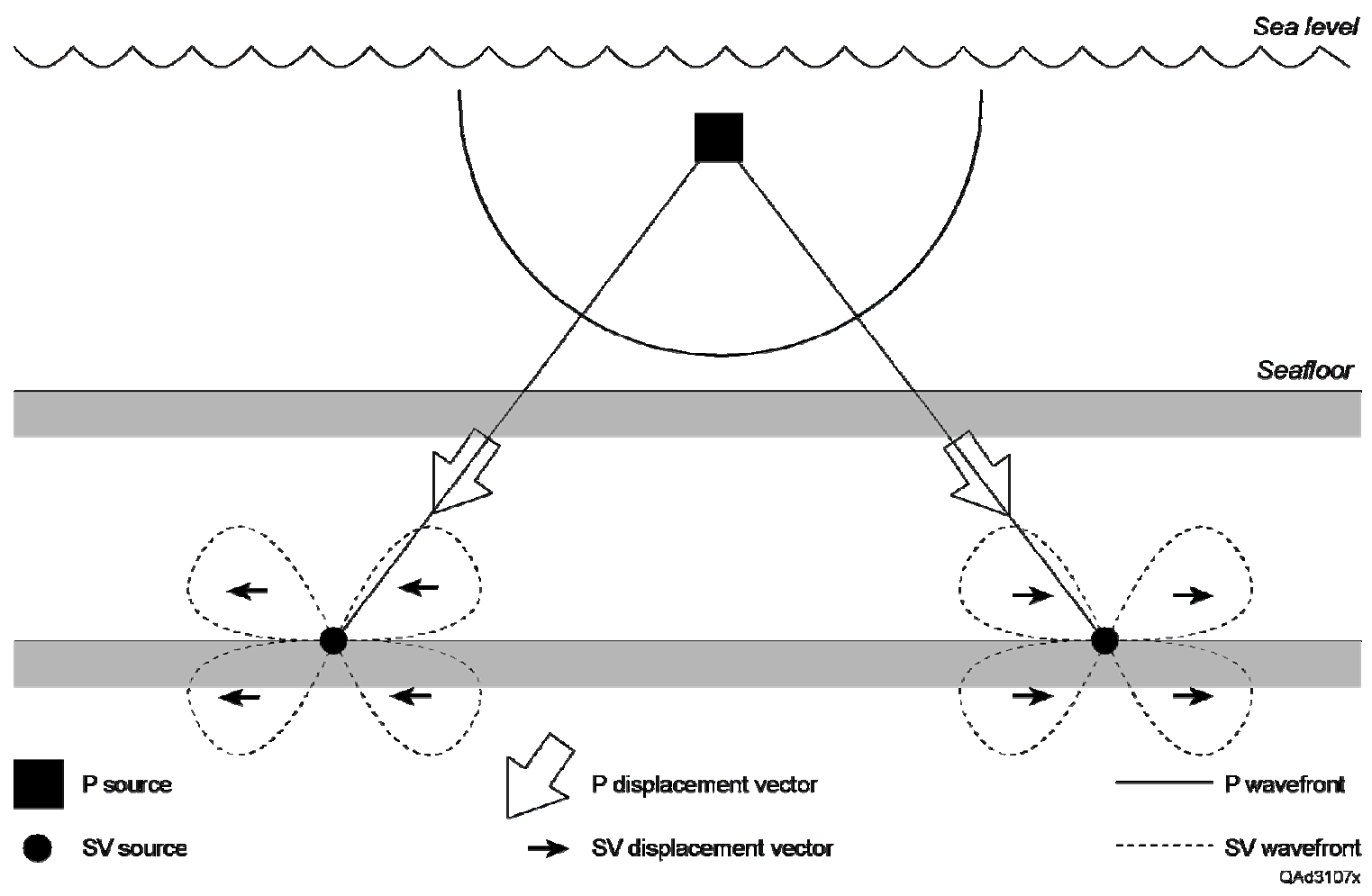

Figure 22. Section view of P-SV radiation pattern. 


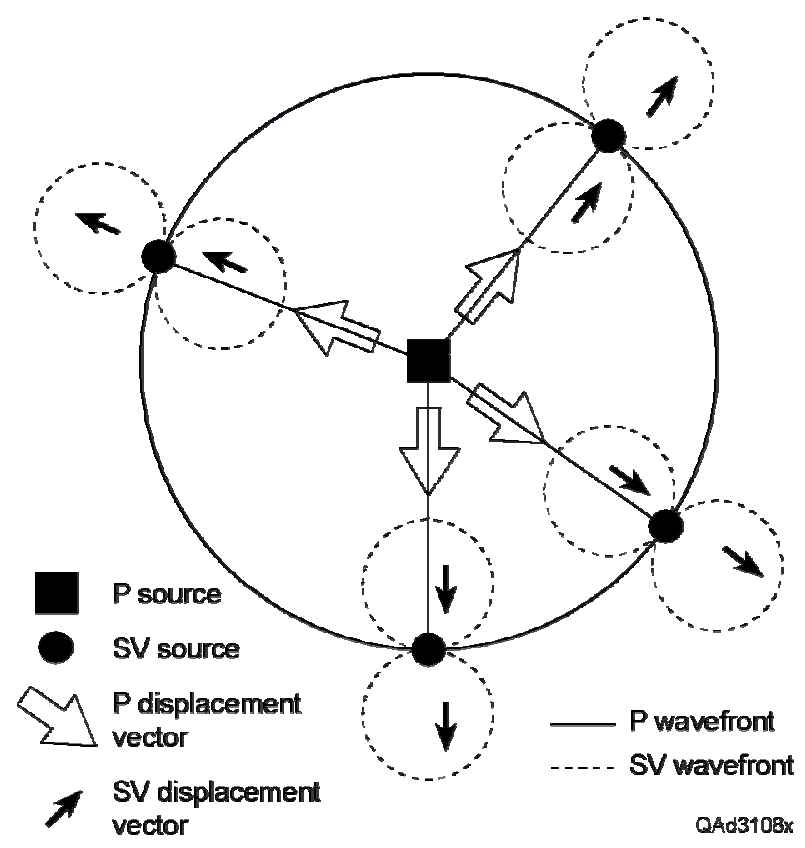

Figure 23. Map view of P-SV illumination pattern.

Distinctions between 3-C and 9-C S-wave target illuminations are easier to visualize if SV and SH radiation patterns associated with each type of data are viewed in a side-by-side format as in Figure 24. These radiation patterns are descriptive of S-wave propagation in an isotropic Earth, not an anisotropic Earth. Analysis of these illumination behaviors leads to several conclusions.

1. A 3-C, scalar, P-wave source generates only an SV S-wave mode. A 9-C horizontal-displacement source creates both SH and SV modes.

2. An SH S-wave mode can be created by only an SH source, which by definition is a 9-C horizontal-displacement source.

3. A 9-C horizontal-displacement source creates SH and SV modes in the Earth volume immediately around its surface-station coordinates. A 3-C, scalar, Pwave source creates a converted-SV mode at subsurface coordinates remote from the source station.

4. In 9-C illumination, all SH and SV particle-displacement vectors throughout the propagation medium are oriented in the same direction as the horizontal source-displacement vector that created the modes. In 3-C illumination, orientation of the SV particle-displacement vector varies with azimuth direction away from the source station. 
5. In 9-C target illumination, SH and SV particle-displacement vectors have a constant algebraic sign (polarity) throughout the propagation medium. In 3-C illumination, the particle-displacement vector of the converted-SV mode has an opposite algebraic sign (polarity) for any two propagation azimuths that differ by $180^{\circ}$.

6. In 9-C data acquisition, SH and SV modes illuminate the subsurface with a different intensity in each azimuth direction. In 3-C data acquisition, the converted-SV mode illuminates the subsurface with the same intensity in all azimuth directions.
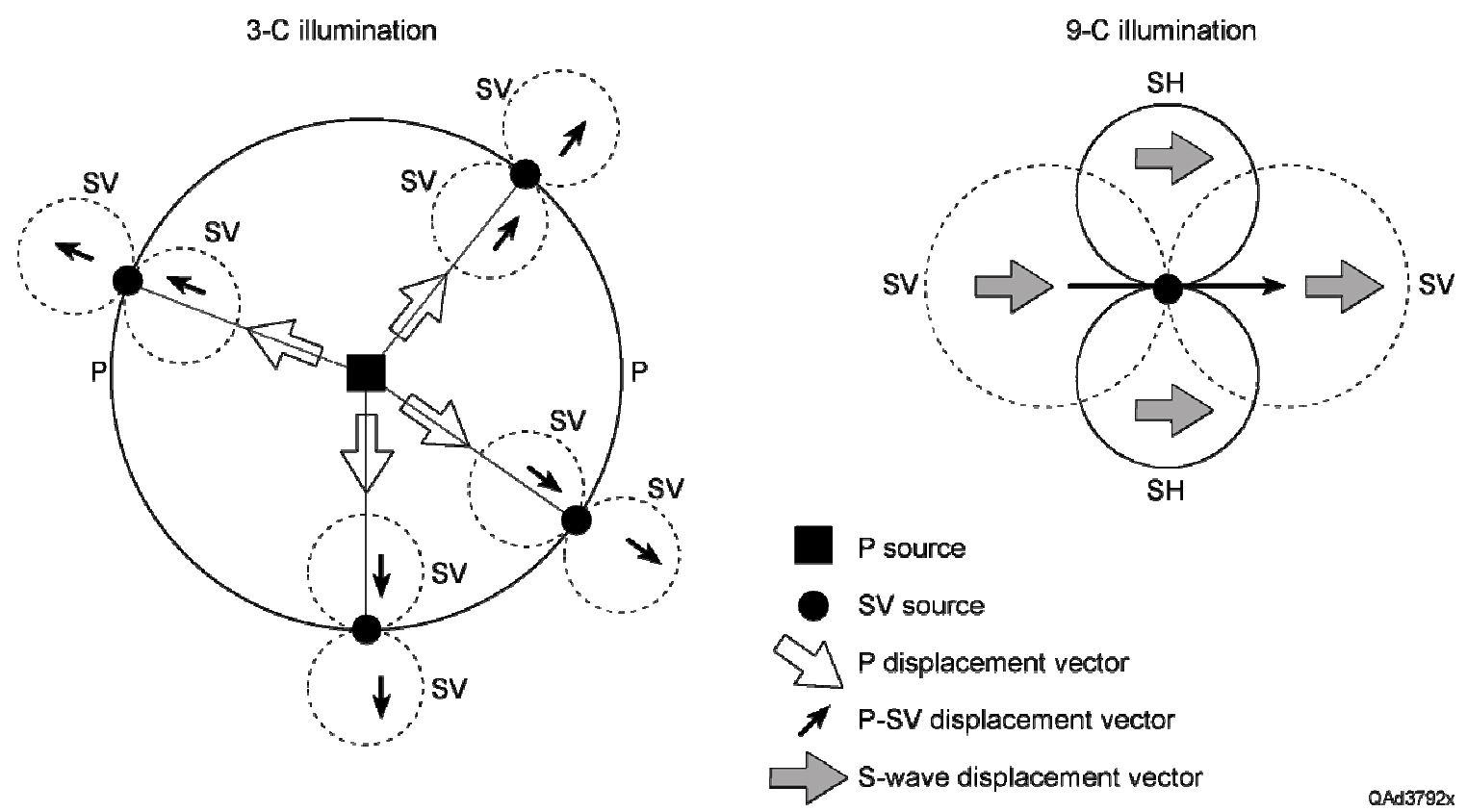

Figure 24. Side-by-side comparison of 3-C and 9-C S-wave illumination patterns.

A final observation about 3-C and 9-C S-wave illumination is based on the principles shown in Figure 25. This diagram illustrates distinctions between the polarizations of SV modes in 3-C and 9-C seismic data, as seen in map view around a source station. SH-mode polarization is not included in the illustration because a 3-C source cannot create an SH mode. For each source, polarization behavior of the SV mode is defined in terms of inline and crossline vector components in each of the four quadrants that encircle the source position. When a P-wave source occupies the source 
station, its downgoing-P wavefield illuminates all four quadrants with equal intensity

(Figs. 23 and 24). However, inline and crossline vector sensors measure a different

polarization for one or both of the horizontal, P-generated, SV displacements in each quadrant, as illustrated in the left diagram. A single horizontal-displacement source will not illuminate all four quadrants around a source station with equal intensity (Figs. 21 and 24). Two orthogonal horizontal-displacement sources must therefore occupy a source station in 9-C data acquisition to create equivalent SV (and SH) illumination intensity throughout the propagation medium. These orthogonal sources create the same SV polarization in all quadrants (right diagram), which is significantly different from 3-C SV polarization behavior.
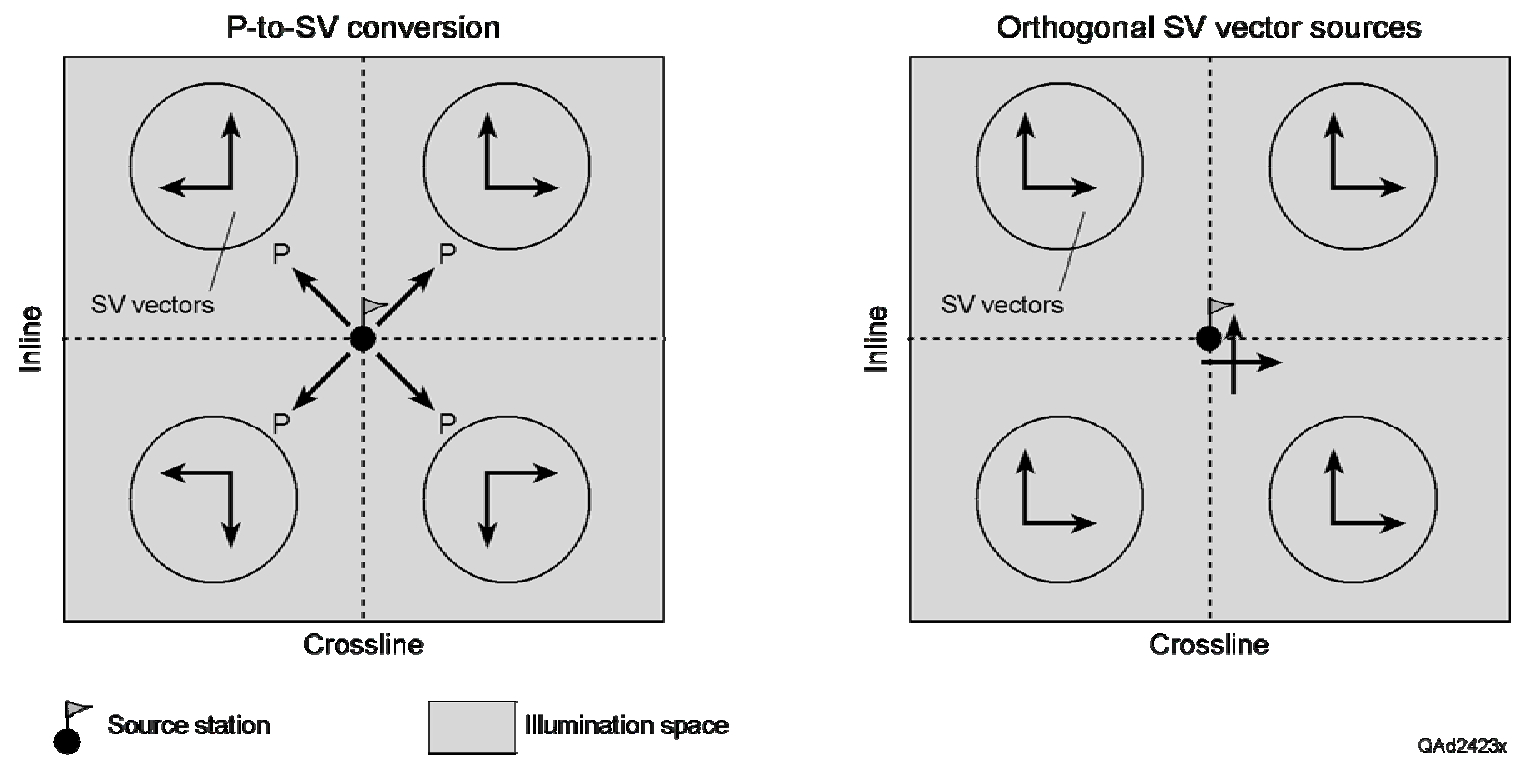

Figure 25. Distinctions between 3-C (left) SV-mode polarization and 9-C (right) SVmode polarization.

\section{Elastic-Wavefield Seismic Stratigraphy}

Multicomponent seismic data, whether 9-C or 3-C data, provide an important new method for interpreting subsurface geology called elastic-wavefield seismic 
stratigraphy. The fundamentals of this interpretation technique are discussed here because several concepts documented in this report are critical to this emerging technology. First, several key terms used in the methodology must be defined. A seismic sequence a succession of relatively conformable seismic reflections bounded by unconformities or their correlative conformities (Mitchum, 1977). The bounding surface of a seismic sequence commonly occurs as a horizon that follows a trend of reflection terminations. A seismic facies is defined as any seismic attribute that distinguishes one succession of reflections from another succession of seismic reflections (Mitchum, 1977). The science of seismic stratigraphy is based on recognizing seismic sequences and seismic facies and then using the spatial geometries, arrangements, and distributions of these sequences and facies to infer depositional environments and lithofacies patterns. The concepts of seismic stratigraphy have dominated the science of seismic interpretation ever since the fundamentals of seismic stratigraphy were made public by Exxon researchers in the mid-1970's (Payton, 1977).

Historical, or traditional, seismic stratigraphy is based on P-P seismic data. Multicomponent seismic data now expand seismic stratigraphy into a new science referred to as elastic-wavefield seismic stratigraphy. The basic premise of elasticwavefield seismic stratigraphy is that any mode of a multicomponent seismic wavefield may provide unique seismic sequence information and/or unique seismic facies information across some stratigraphic intervals that cannot be observed in the other modes of the wavefield. Seismic stratigraphy analyses now do not need to be limited to P-P data, as they have for decades. 
The logic for the fundamental premise of elastic-wavefield seismic stratigraphy is based on the principles discussed in the preceding sections. First, the particledisplacement vectors of a multicomponent seismic wavefield test the properties of the Earth in different directions (Fig. 1). As a result, the displacement vector of one mode may detect seismic facies (Earth fabric) that are different from what other displacement vectors detect and may be affected by stratal surfaces that are different from those that affect the displacement vectors of other modes. Second, each wave mode illuminates a target with a unique radiation pattern geometry, which may cause one mode to reveal a target feature not seen with other modes. Third, all wave modes have distinct reflectivity behaviors at an Earth interface, which sometimes causes one mode to emphasize a suite of stratigraphic interfaces differently than do its companion modes.

If 9-C seismic data are used, Earth fabric can be measured using five independent particle-displacement vectors (P-P, SH-SH, SV-SV, P-SV, SV-P). If 3-C seismic data are used, Earth fabric can be tested using only two particle-displacement vectors (P-P and P-SV). The increased number of independent fabric-sensing displacement vectors associated with 9-C seismic data leads to the conclusion that more rock, fluid, and general Earth-fabric information should be provided by 9-C seismic data than by 3-C data. Similarly there is a greater likelihood that 9-C seismic data can image a stratal surface that is not imaged by 3-C data, and that 9-C data can reveal a seismic sequence or a seismic facies that is not revealed by 3-C data. 


\section{DATA-PROCESSING DIFFERENCES BETWEEN 9-C AND 3-C S-WAVE DATA}

Software that transforms S-wave modes of 9-C seismic data into S images differs in fundamental ways from software that produces S-wave images from 3-C data. Common-midpoint (CMP) data-processing concepts, which have been used in oil and gas applications for decades, can be used to produce S-wave images from 9-C data. A different data-processing strategy called common-conversion-point (CCP) imaging is required for constructing S-wave images from 3-C data. Key differences between these two data-processing technologies (CMP and CCP) are described in this section.

\section{Common Midpoint (CMP) Imaging}

The basic requirement for CMP imaging is that the propagation velocity of the reflected, upgoing wavefield be the same as the propagation velocity of the downgoing, illuminating wavefield. In the P-P seismic imaging that the oil/gas industry has done for approximately 50 years, downgoing and upgoing wavefields both travel at P-wave velocity Vp. CMP software was developed originally to make only P-P images and has been used for this restricted, seismic-mode imaging until recently. However, CMP imaging can be applied in any situation in which the downgoing and upgoing wavefields have equivalent propagation velocities. Thus, when 9-C data are acquired, $\mathrm{SH}-\mathrm{SH}$ and SV-SV images, in addition to P-P images, can be made with CMP software. Downgoingand upgoing-SH wavefields that travel with velocity $\mathrm{V}_{\mathrm{SH}}$ are segregated from the 9-C wavefield and are used to make an SH-SH image. Downgoing- and upgoing-SV wavefields that travel with velocity Vsv, which differs slightly from $\mathrm{SH}$ velocity $\mathrm{V}_{\mathrm{SH}}$, are then extracted from the 9-C data and used to make an SV-SV image. Many versions of 
CMP software are available throughout the seismic data-processing industry. Any of these software packages can be used to process 9-C seismic data to create SH-SH and SV-SV shear-wave images, in addition to the standard P-P compressional-wave image that has been made for decades. The raypaths involved in CMP imaging are shown in Figure 26. This diagram shows that in a flat-layered Earth, CMP reflection points generated at different reflector depths stack vertically above each other at coordinate Xm, the common midpoint, located halfway between the source station and the receiver station. The image-point trend labeled CCP is discussed in the following section.

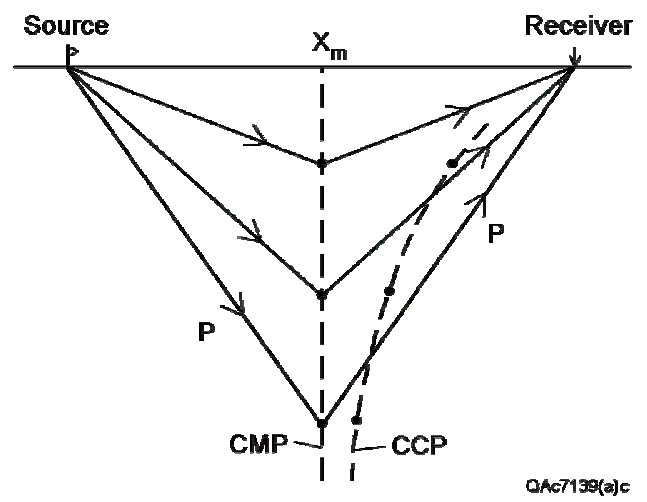

Figure 26. Distinction between 9-C CMP image points (vertical dash line) and 3-C CCP image points (curved dash line). Raypaths show the propagation paths involved in CMP imaging.

\section{Common-Conversion-Point (CCP) Imaging}

Common-midpoint (CMP) imaging concepts cannot be used when the propagation velocity of the downgoing, illuminating wavefield differs from the propagation velocity of the upgoing reflected wavefield. The most common situation where this wave physics is encountered involves the P-SV mode, which is created when a 
downgoing-P illumination wavefield converts to an upgoing-, reflected-SV wavefield via P-to-SV mode conversion at a reflecting interface. As has been stressed in the preceding sections, a P-SV mode is the only S-wave mode that can be extracted from 3-C seismic data. The inverse mode, SV-P, which is created by a downgoing-, illuminating-SV wavefield converting to an upgoing-, reflected-P wavefield via SV-to-P mode conversion at a reflecting interface, is another situation where CMP data-processing concepts cannot be used. An SV-P mode is available only with 9-C seismic data because an SV source is required to produce the downgoing illumination wavefield.

For each of these converted-S modes (P-SV and SV-P), the image point does not occur at common-midpoint coordinate Xm, as in CMP imaging. In 3-C (or 4-C) P-SV imaging, the downgoing wavefield has a faster velocity (Vp) than the upgoing wavefield (Vsv). As a consequence of Snell's law, the image point does not occur at midpoint Xm but at a coordinate that is closer to the receiver station than to the source station. This image coordinate is called the common-conversion point (CCP). The raypaths involved in CCP imaging of a P-SV mode are depicted in Figure 27. This diagram shows that in a flat-layered Earth, CCP image points generated at different depths do not stack vertically above each other, as do CMP image points, but move closer to the receiver station as reflecting interfaces are imaged nearer the Earth surface. 


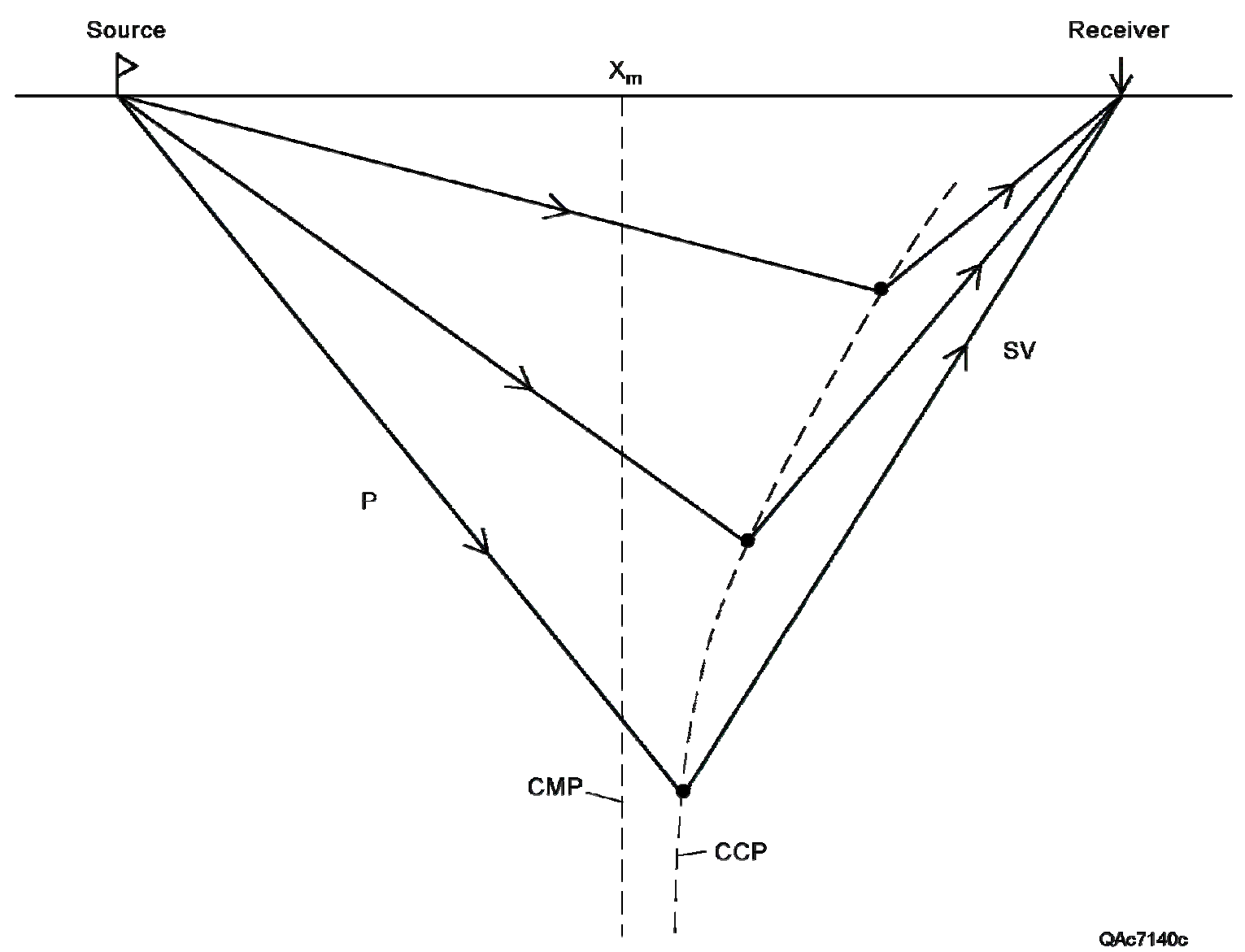

Figure 27. Distinction between 3-C CCP image coordinates (curved dash line) and 9-C CMP image point coordinates (vertical dash line). Raypaths illustrate propagation paths involved in CCP imaging.

In 9-C SV-P imaging, the downgoing wavefield propagates at a velocity (Vsv) slower than that of the upgoing wavefield (Vp). Now as a result of Snell's law, the image point occurs closer to the source station than to the receiver station. This image point is still called a common-conversion point even though it is located at a subsurface coordinate different from the CCP coordinate associated with 3-C P-SV imaging. The raypath involved in 9-C CCP imaging of SV-P data is illustrated in Figure 28. Again, the image points generated at different depths will not stack vertically above each other. In 
contrast to 3-C P-SV imaging, SV-P image coordinates move closer to the source station, not the receiver station, as reflecting interfaces approach the Earth’s surface.

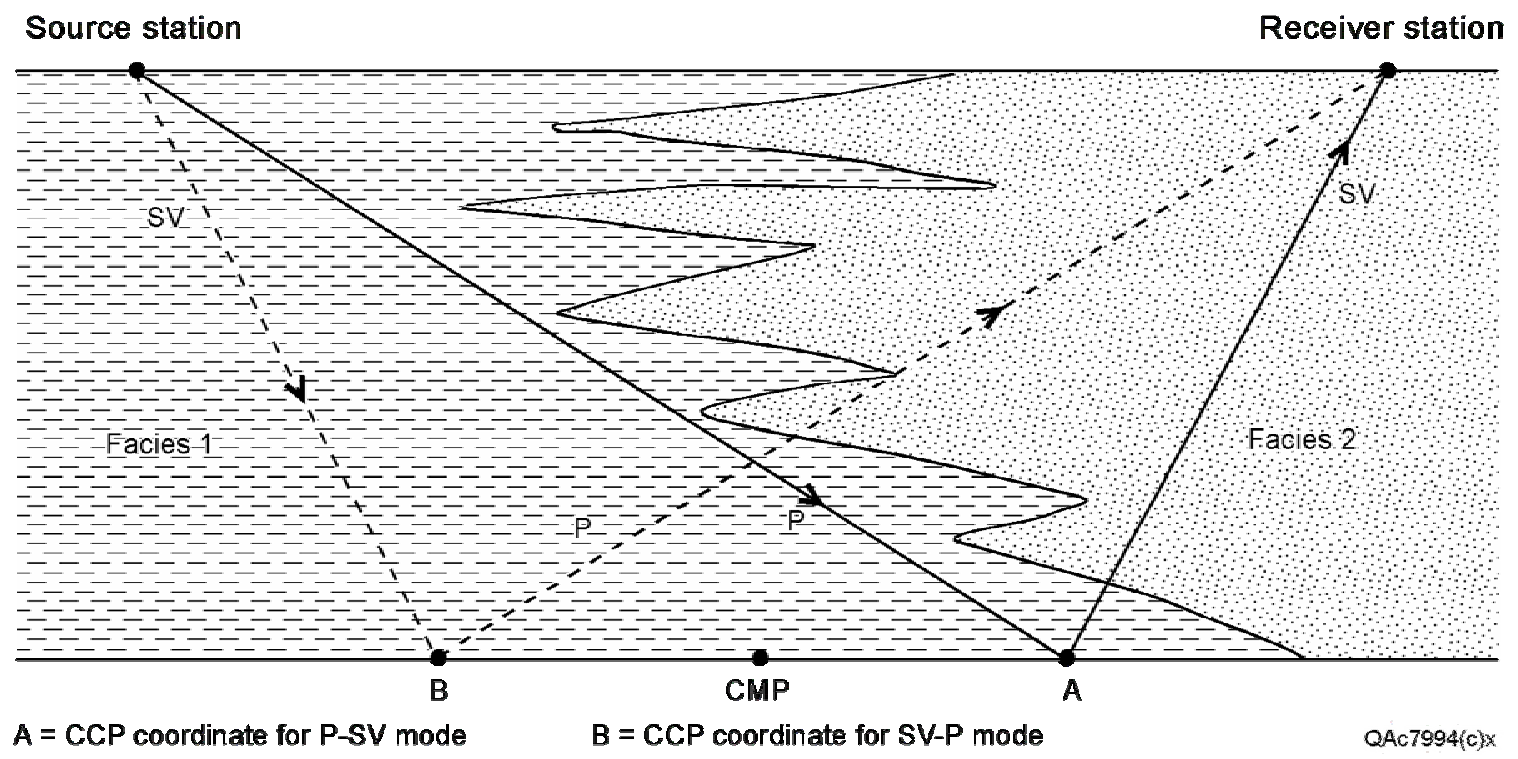

Figure 28. Distinction between a 9-C SV-P CCP raypath (dash line) and a 3-C P-SV CCP raypath (solid line).

\section{CMP and CCP Velocity Analyses}

The stacking and migration velocities needed for 9-C (CMP) and 3-C (CCP) Swave imaging have to be determined by different analytical procedures. The fundamental reason that an approach to velocity estimation has to be done for 9-C data that is different from that for 3-C data can be explained by referring to the simple Earth model in Figure 29. In this model there is a change in rock facies along the imaging raypaths. P-wave velocity Vp and SV velocity Vsv in Facies 1 are assumed to be different from the values of Vp and Vsv in Facies 2. 


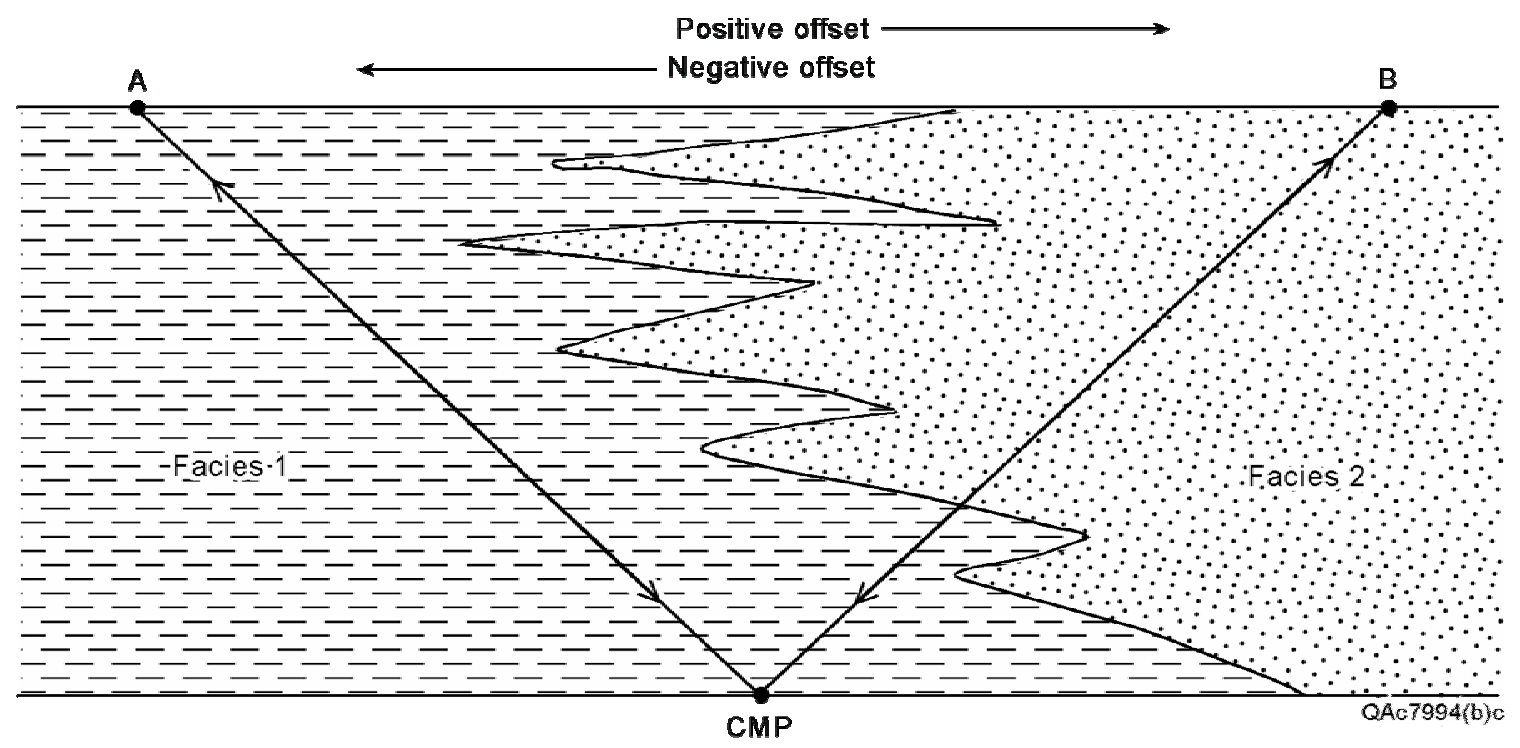

Figure 29. Traveltimes for positive offsets are the same as traveltimes for negative offsets in 9-C CMP imaging because the lengths of the travel paths in Facies 1 and 2 are the same for both offset options.

The offset between source and receiver stations now has to be defined in terms of the direction that the raypath takes to propagate from the source to the receiver. A receiver offset to the right of the source will be defined arbitrarily as a positive offset; receivers to the left of the source station will then be in the negative offset direction. In 9C CMP S-wave imaging (Fig. 29), the same raypath velocity and traveltime occur in both negative and positive offset directions because the lengths of the travel paths in Facies 1 and in Facies 2 are the same when B is the source station and $\mathrm{A}$ is the receiver station (negative offset) as they are when $\mathrm{A}$ is the source station and B is the receiver station (positive offset). The same stacking and migration velocities are therefore calculated in positive and negative offset directions when 9-C SH-SH and SV-SV data are processed. 
A different conclusion is reached in 3-C P-SV CCP velocity analysis. The raypaths involved in 3-C CCP imaging of the P-SV mode are shown in Figure 30. If A is the source station and B is the receiver station (positive offset), the velocity of the downgoing-P mode is controlled by Facies 1 and the upgoing-SV-mode velocity is determined by Facies 2. The image coordinate is $\mathrm{CCP}_{\mathrm{A}}$. When $\mathrm{B}$ is the source station and $\mathrm{A}$ is the receiver station (negative offset), most of the P-wave velocity is controlled by Facies 2, and all of the upgoing-SV raypath is in Facies 1. The image coordinate is now $\mathrm{CCP}_{\mathrm{B}}$. Assuming that velocities Vp and Vsv in Facies 1 differ from those of Vp and Vsv in Facies 2, CCP stacking and migration velocities calculated for positive offsets and negative offsets are not the same. That different velocity behaviors are observed in opposite offset directions for 3-C P-SV imaging is a fundamental distinction between the wave physics of 9-C and 3-C seismic data.

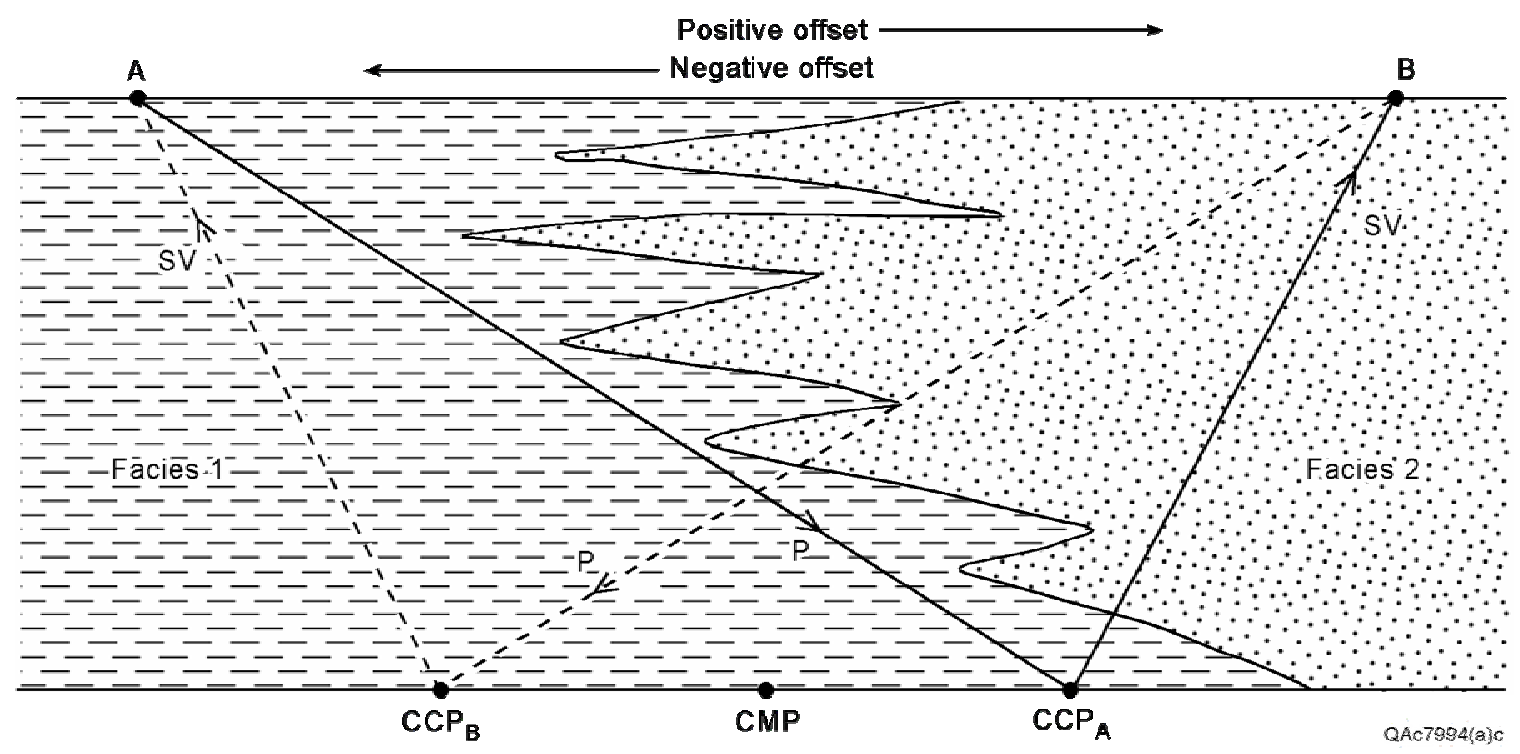

Figure 30. Traveltimes for positive offsets are not the same as traveltimes for negative offsets in 3-C CCP imaging because the lengths of the P and SV raypaths in Facies 1 and 2 change when the offset direction changes. 


\section{CMP and CCP Stacking}

For a seismic image to be created, the image space between all source and receiver pairs is segregated into small subvolumes called stacking bins. During data processing, data traces are positioned across this image space by calculating the bin locations where successive image points occur. In CMP (9-C) imaging in a flat-layered Earth, image points occur at the midpoint between source and receiver regardless of the depth of the reflecting interface (Fig. 26). A CMP trace is shifted in time (source-static correction, receiver-static correction, other static corrections, and normal moveout correction), and then the entire data trace is positioned vertically at the common midpoint for the source-receiver pair that produced the trace. This type of imaging is indicated in Figure 31 by the vertical data trace in stacking-bin column A located at the common midpoint for the indicated source and receiver. That data trace is created at the indicated source station and recorded at the labeled receiver station. In CMP image space, however, the trace is positioned in stacking bin A at the midpoint between source and receiver coordinates. 


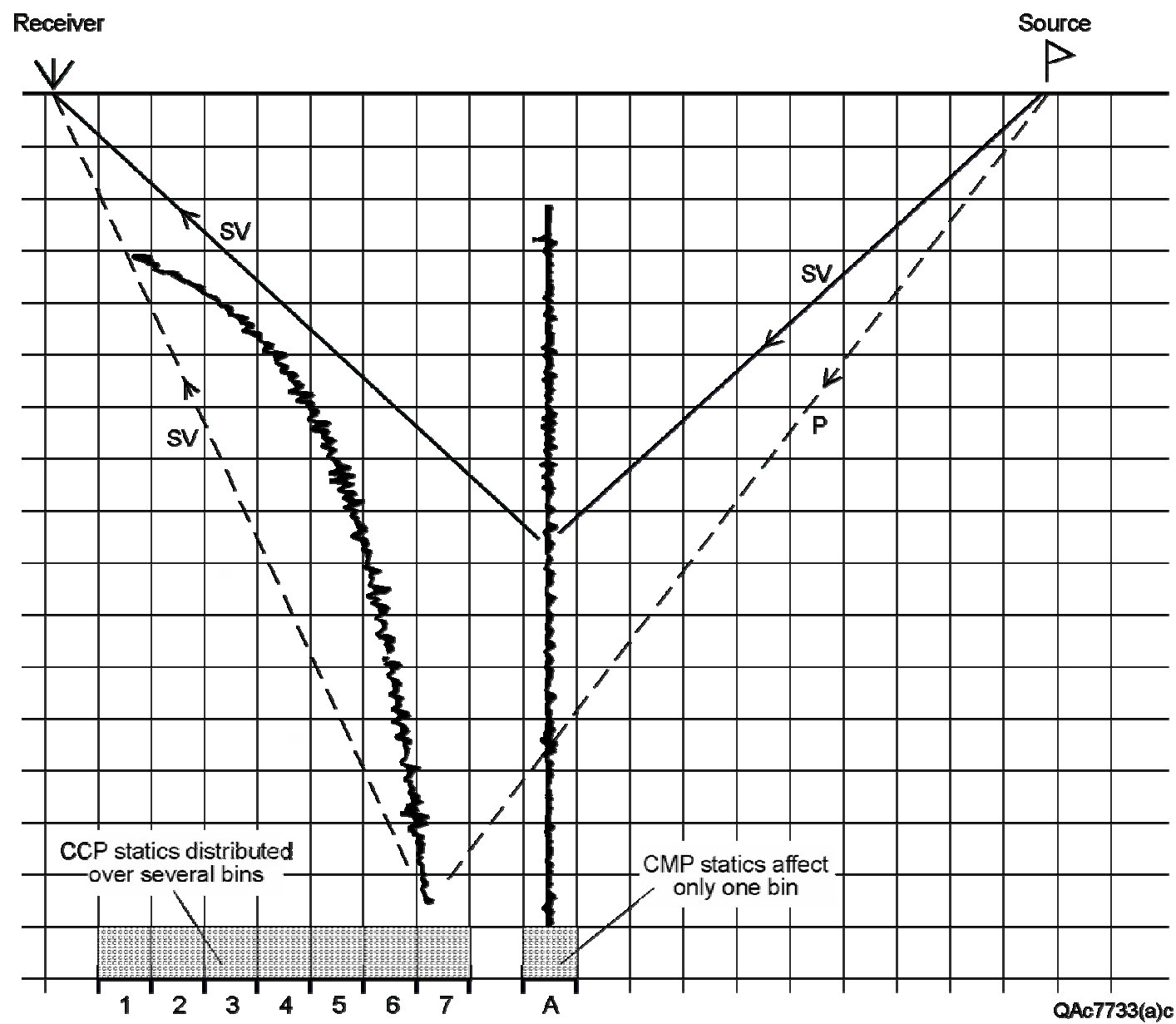

Figure 31. Comparison of 9-C CMP image trace (vertical in stacking bin A) and 3-C CCP image trace (curved across stacking bins 1 through 7).

Robust CMP stacking algorithms are widespread across the seismic industry, and most commercial seismic data-processing shops have extensive experience in CMP processing. Numerous seismic data-processing companies can therefore create goodquality SH-SH and SV-SV images from 9-C data because CMP concepts that they understand and have applied countless times are all that are required to create these Swave image options. The basic requirement is that a horizontal-displacement vector 
source be positioned at the source station to create downgoing-SH and -SV illumination modes. The raypath notation in Figure 31 indicates only downgoing- and upgoing-SV modes because the objective is to distinguish between SV-SV and P-SV imaging.

The curved wiggle trace in Figure 31 shows where the data trace would be distributed across the image space if a P-wave source occupied the source station, a 3-C vector sensor occupied the receiver station, and the data were acquired according to 3-C P-SV imaging constraints. In this case, the downgoing raypath is a $\mathrm{P}$ wave, and the upgoing raypath is an SV mode. Now the static and normal-moveout time adjustments made to the image trace affect data in several columns of stacking bins. Segments from several CCP traces have to be patched together to create a vertically stacked trace in each column of stacking bins. For example, three 3-C CCP-processed data traces that are offset from each other by one bin dimension in seismic image space are shown in Figure 32. That part of trace A between points 1 and 2 has to be combined with the data window extending from 2 to 3 in trace B and with the data window extending between points 3 and 4 in trace $C$ to create a vertical wiggle trace extending from point 1 to point 4 in the shaded column of stacking bins. 3-C CCP stacking is thus fundamentally different from 9-C CMP stacking. As a consequence of the more complex requirements of CCP stacking, some seismic data-processing shops do not have software or experience needed to do 3-C P-SV imaging. Even data-processing shops that have established themselves as reputable CCP data imagers are still developing some critical software and improving older algorithms. 


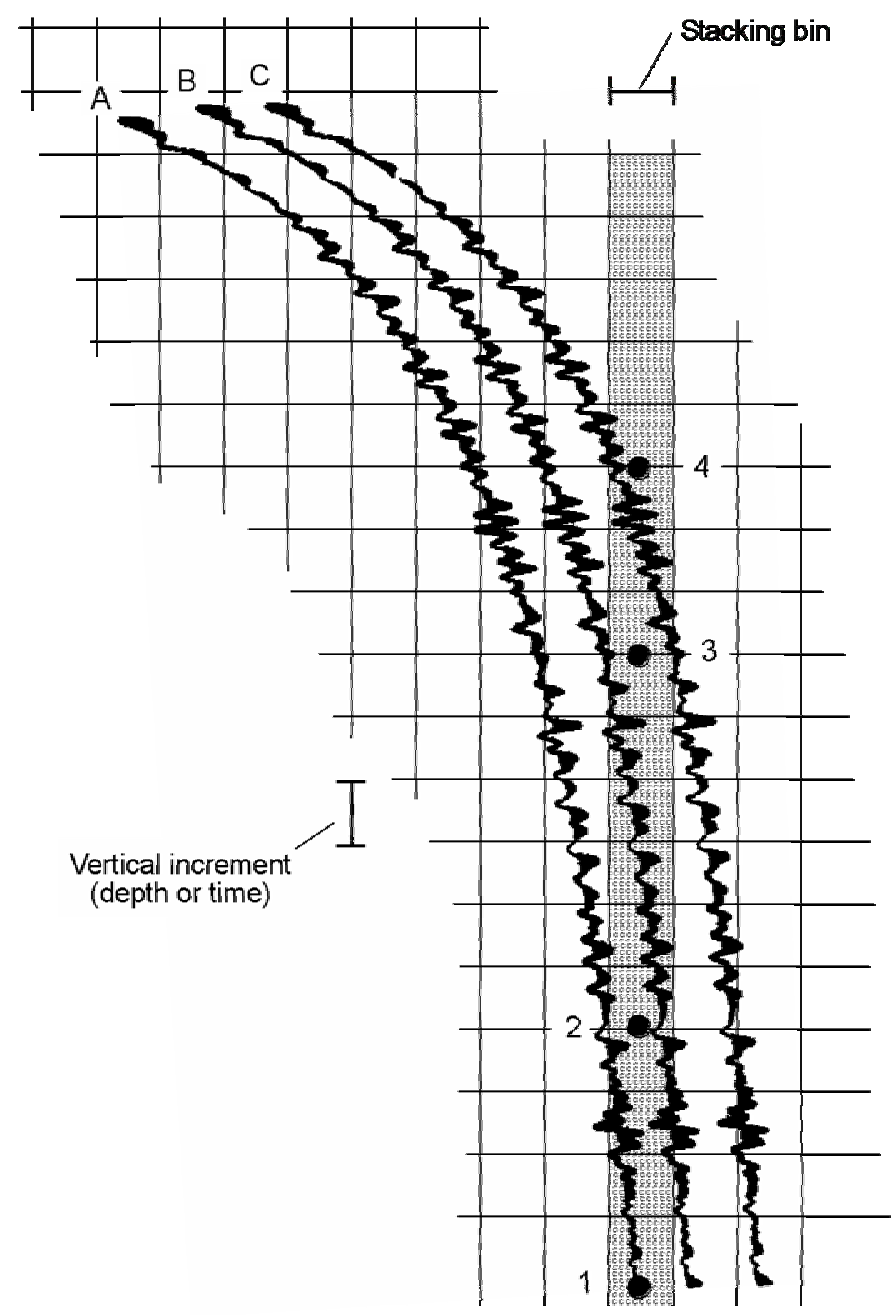

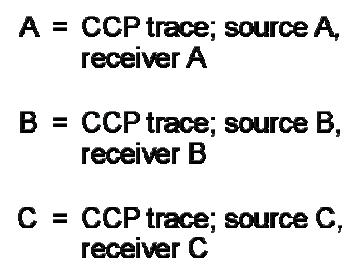

1, 2 = Data segment from trace $A$

2, 3 = Data segment from trace $B$

3, 4 = Data segment from trace C

Figure 32. Single vertical image trace in one stacking bin of CCP image space (shaded column) must be constructed by summing data from different time windows of all CCP traces that traverse the bin.

The parameter that controls the curvature of a CCP trace in CCP-image space is the $\mathrm{Vp} / \mathrm{Vs}$ velocity ratio in the propagation medium. A model that illustrates this fact for small angles of incidence is presented as Figure 33. This simple, straight-raypath model shows that a 3-C P-SV image coordinate is defined by offsets $\mathrm{Xp}$ and $\mathrm{X}_{\mathrm{SV}}$ from the source and receiver stations and that these offsets are proportional to the $\mathrm{Vp} / \mathrm{Vs}$ velocity 
ratio in the propagation medium. The top equation listed in this illustration is Snell's law of reflection, the middle equation is a statement of the raypath geometry shown in the model, and the bottom equation is valid when the incident angles are small enough that sine is the same as tangent. For larger angles of incidence and reflection in a layered Earth, the relationship between CCP image coordinates and the Vp/Vs velocity ratio is more complicated than the simple equation in Figure 33. In real Earth media, a key requirement of 3-C CCP processing is to create accurate $\mathrm{Vp} / \mathrm{Vs}$ imaging functions across seismic image space by first stacking P-SV data with a large number of $\mathrm{Vp} / \mathrm{Vs}$ values and then determining which $\mathrm{Vp} / \mathrm{Vs}$ value produces optimal-quality stacked data at each image coordinate. The concept is similar to the time-variant, space-variant, velocity-semblance technology that is used to stack CMP data.

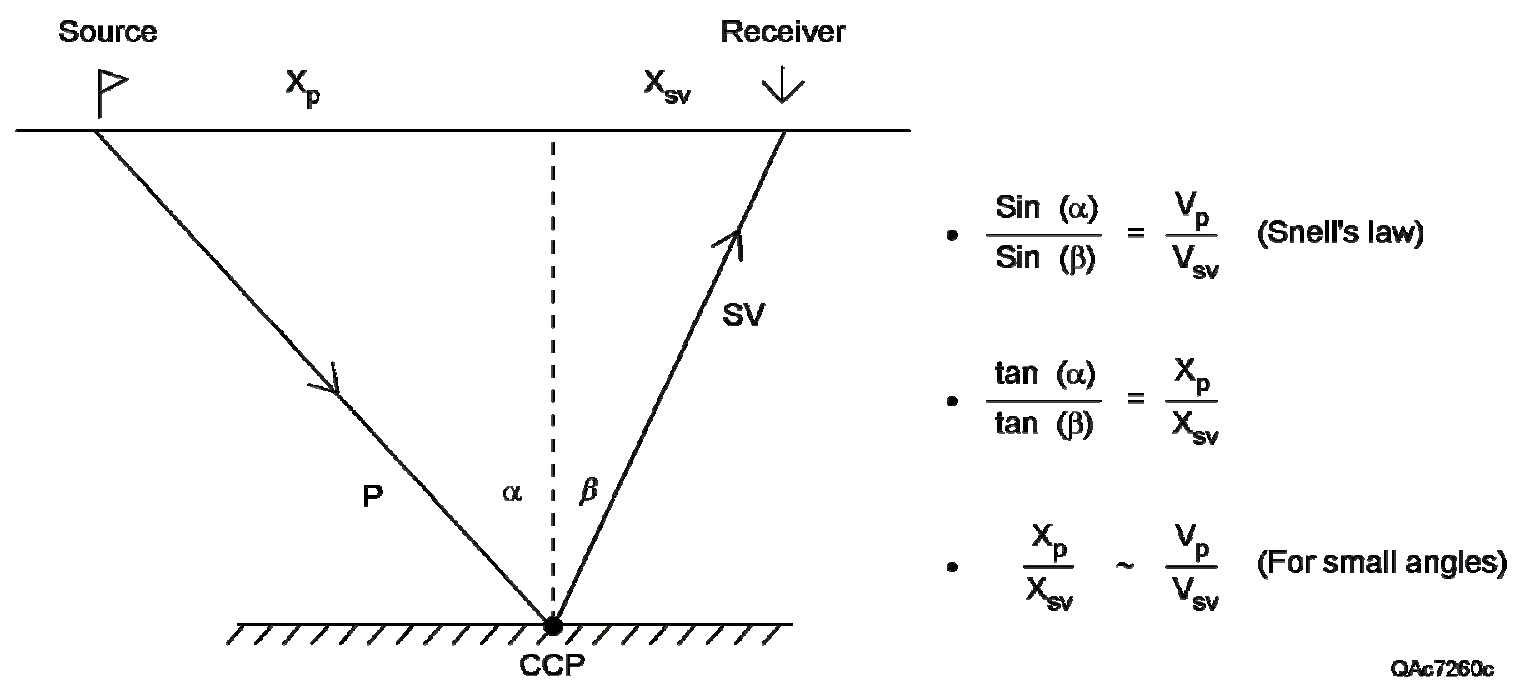

Figure 33. Simple, straight-raypath model showing that the velocity ratio $\mathrm{Vp} / \mathrm{Vs}$ in the propagation medium controls the position of a 3-C P-SV image coordinate in seismic image space. 
Although construction of 3-C P-SV images concentrates on determining accurate values of $\mathrm{Vp} / \mathrm{Vs}$ over the total image space, some data processors take shortcuts. The most common shortcut is to do asymptotic binning. In asymptotic binning, the CCP coordinate for the deep part of the image space, where the CCP image trace is almost vertical (Figs. 31 and 32), is calculated, and then the entire data trace is assumed to be vertically aligned at that coordinate. This approximation would cause all of the curved trace in Figure 31 to be positioned in stacking bin 7, the asymptotic bin for that trace. The deep part of the image would be correct, but the upper part would be incorrect, with the imaging error increasing as the reflecting interface approaches the Earth’s surface. For deep targets, asymptotic binning is acceptable. For shallow targets, it is not.

More advanced data-processing shops have abandoned asymptotic binning and replaced that shortcut technique with procedures that calculate time-dependent and spacedependent estimates of $\mathrm{Vp} / \mathrm{Vs}$ over the total CCP image space. In so doing, however, they still often take shortcuts, such as giving little attention to determining accurate values of $\mathrm{Vp} / \mathrm{Vs}$ in shallow data windows if there is no exploration interest in shallow targets. This imaging philosophy is a practical procedure in the low-profit-margin business of seismic data processing. There is no financial reward for work done to make the shallowest part of a CCP image correct if no one is interested in shallow geology. 


\section{EXAMPLES OF 9-C AND 3-C SEISMIC DATA}

Data from several multicomponent seismic surveys will be presented in this section to illustrate selected concepts that distinguish 9-C S-wave data from 3-C S-wave data. The ideal way to evaluate distinctions between 9-C and 3-C seismic data is to have both 9-C and 3-C data acquired at the same location. However, two separate seismic surveys are required to satisfy this same-location objective without biasing the analysis of the data toward one or the other of the multicomponent technologies. Such bias will likely occur if analysis is limited to a single seismic survey because 9-C CMP imaging geometry differs from optimal 3-C CCP imaging geometry (Figs. 26, 27, 29, and 30). Source-receiver geometries that result in high, uniform stacking fold of 9-C CMP data rarely produce optimal image-fold conditions for 3-C CCP data. Similarly, most 3-C seismic survey geometries are not optimal for 9-C CMP data acquisition. These comments should not be construed to mean that a single seismic acquisition program cannot be designed that will produce optimal-quality data for both 9-C and 3-C imaging. We think that as multicomponent seismic technology gains acceptance, such surveys will be done. The real-world situation for this study, however, was that no single survey using a geometry that was optimal for both 9-C and 3-C data was available for our analysis. We are not aware that such a survey exists anywhere.

We began this project with the rather naïve assumption that a 9C3D seismic survey we called the Ashland Survey would be an ideal database for comparing 9-C and 3-C data. However, as we pursued the study we came to the conclusion that because the Ashland Survey geometry was designed for CMP imaging, our findings would probably 
be biased toward the advantages of SH-SH and SV-SV CMP modes, and the P-SV CCP mode would not be judged on a fair basis. We thus decided to satisfy our research objectives by explaining distinctions between 9-C and 3-C seismic data from theoretical and data-processing points of view and then showing S-wave data acquired using surveys in which the acquisition geometry was optimal for either 9-C or 3-C data, but not for both. That decision dictated the content and format of this report.

\section{Distinctions between SH-SH and SV-SV S-Wave Modes}

The fundamental thesis of this investigation is that several S-wave modes can be extracted from multicomponent seismic data and that each of these modes can provide different geologic information about the Earth than can its companion modes. A concept that has been stressed throughout this report is that the greatest differences between Swave modes occur when 3-C P-SV data are compared with 9-C SH-SH and SV-SV data. However, we will depart from this thesis temporarily and start this section with an example that illustrates the principle that even 9-C SH-SH and SV-SV S-wave data differ in fundamental ways.

Data from the Ashland 9C3D seismic survey are displayed in Figure 34. These data are prestack super gathers constructed to show distinctions between SV-SV and SHSH data in field-record format. The data-processing procedures used to transform the 9-C data from field-coordinate data space (where SH-SH and SV-SV modes are mixed together) to the radial-transverse data space used in this display (where SH-SH and SVSV modes are separated) were discussed by Simmons and Backus (1999) and will not be repeated here. 


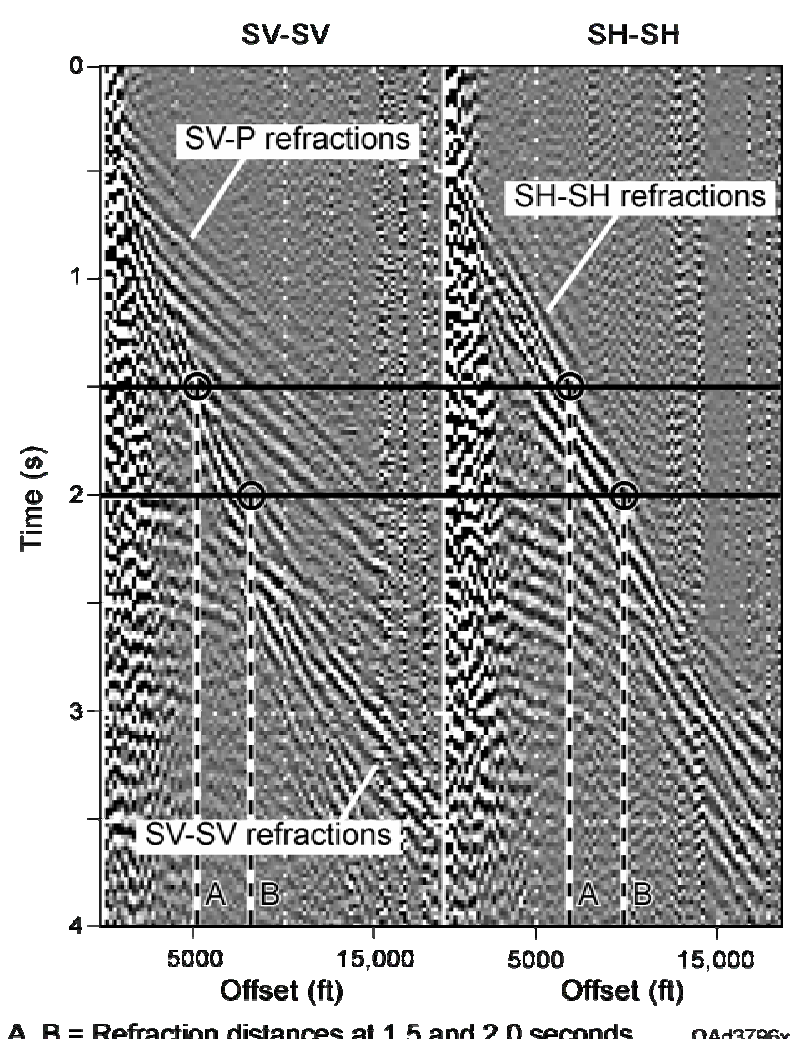

Figure 34. 9-C SV-SV and SH-SH super gathers shown in field-record format (Simmons and Backus, 1999).

Two distinctions between SH-SH and SV-SV shear-wave modes can be illustrated with this data example. First, the downgoing-SV mode converts to two upgoing modes, SV and P, but the downgoing-SH mode converts to only one upgoing mode, $\mathrm{SH}$. This wave physics is demonstrated by the presence of both SV-SV and SV-P refraction events in the SV-SV data but only SH-SH refraction events in the SH-SH data. These refraction events are labeled on the data to ensure that there is no misunderstanding about what is being viewed. We see in this SV-SV field record (left) the exact wavefield behavior described by the SV dual-reflectivity equations in Figures 16, 17, and 19 and in the SH- 
SH field record (right) the behavior indicated by the single-reflectivity equation in Figure 11. The fact that a downgoing-SH mode creates only a reflected-SH mode, but a downgoing-SV mode creates both reflected SV and P is why the SH mode is sometimes called a "pure" shear mode. Said another way, SH-SH field records contain only shearwave information, but SV-SV field records contain both S-wave and P-wave information mixed together in a rather complicated way, as the SV reflectivity equations imply (Fig. 17). This distinction between SH-SH and SV-SV data is fundamental.

The events that curve across the data panels of Figure 34 between 2 and 3 seconds are SV-SV reflection events (left panel) and SH-SH reflection events (right panel). It is rare to see SV-P reflections in field-record formats. None is obvious in the left data panel of the figure. However, SV-P reflections should contaminate the SV-SV reflections in almost the same proportion as the SV-P refractions contaminate the SV-SV refractions.

The second distinction to note between the SV-SV and SH-SH data of Figure 34 is that there is a significant difference in SV and SH propagation velocities. This fact is illustrated by marking the distances A and B that S-wave refraction events propagate after 1.5 and 2 seconds of traveltime. As shown in the figure, distances A and B for the SH-SH data are greater than distances A and B for the SV-SV data. SH velocity parallel to bedding (the direction of refraction travel) is therefore greater than SV velocity.

To further demonstrate differences between SH and SV propagation velocities, the super gathers in Figure 34 are processed to emphasize primary reflection events in the time window between 2 and 2.5 seconds. These processed data are shown as Figure 35. The normal-moveout corrections used to flatten reflection events in both the SH-SH and SV-SV data were done using SH-derived stacking velocities. The SH-SH events are 
flattened, as expected (right panel), but the SV-SV events are overcorrected (left panel).

This test is a compelling demonstration that SH stacking velocities are larger than SV stacking velocities. Because stacking velocities are horizontal velocities, the test also confirms that differences between SH and SV velocities exist at deep interfaces where primary reflections originate, as well as at shallow refracting interfaces (Fig. 34).

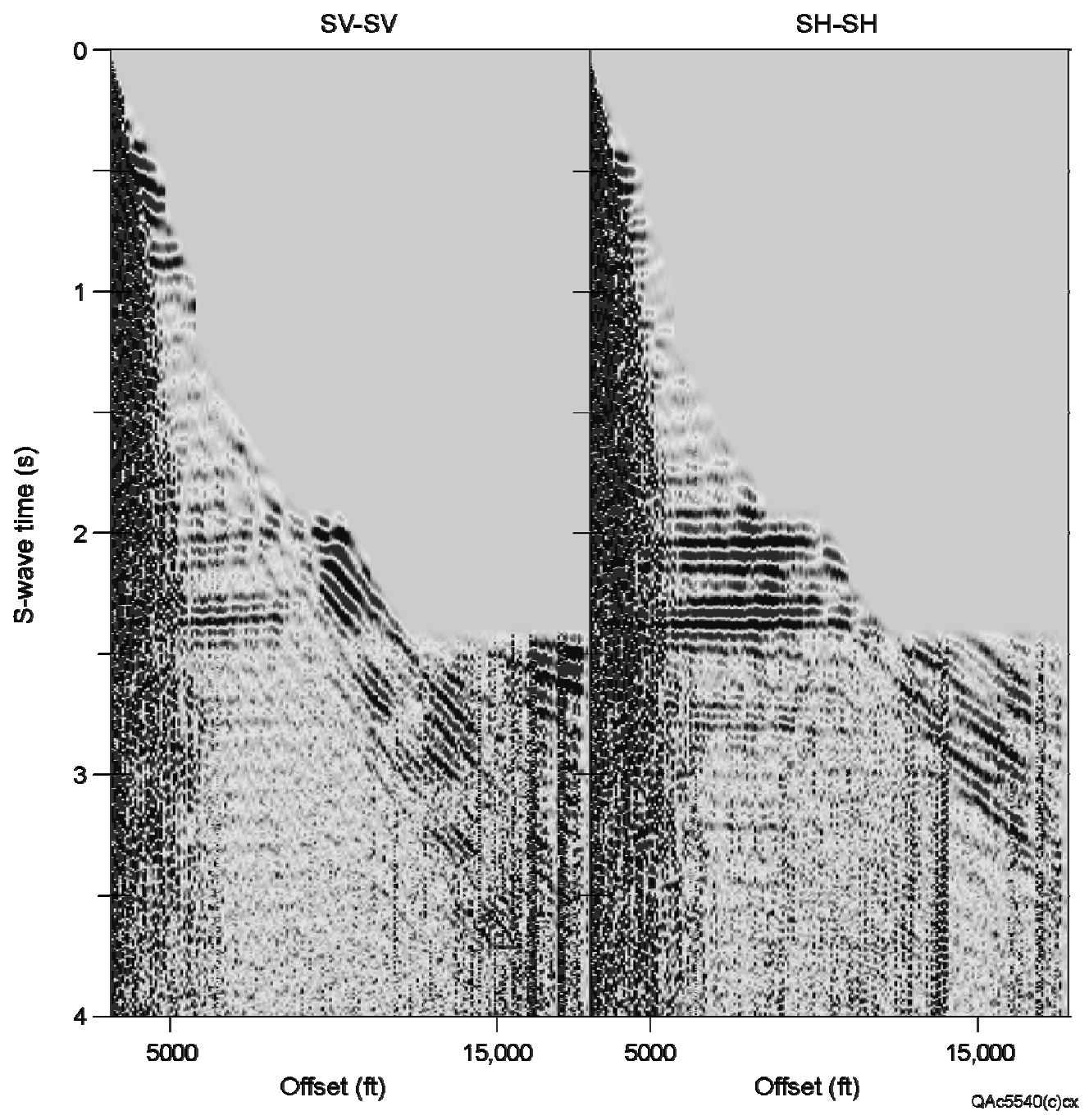

Figure 35. 9-C SV-SV and SH-SH trace gathers after processing to emphasize primary reflections between 2 and $2.5 \mathrm{~s}$. Reflection events in both modes are corrected to horizontal events with SH-derived velocities to demonstrate that SH and SV stacking velocities differ (Simmons and others, 1999). 
Distinctions between SH and SV propagation velocities in a layered Earth were published by Levin $(1979,1980)$ more than 2 decades ago. A part of Levin's work is repeated as Figure 36. This diagram shows that SH and SV velocities are equal when modes travel vertically in a transverse isotropic (flat layered) Earth and when they propagate away from their coincident point of origin at one particular takeoff angle (where velocity curves cross). The velocities differ at all other propagation angles, with the difference being greatest along the horizontal propagation axis. In the horizontal direction, SH velocity is significantly greater than SV velocity, which is what the data in Figures 34 and 35 confirm.

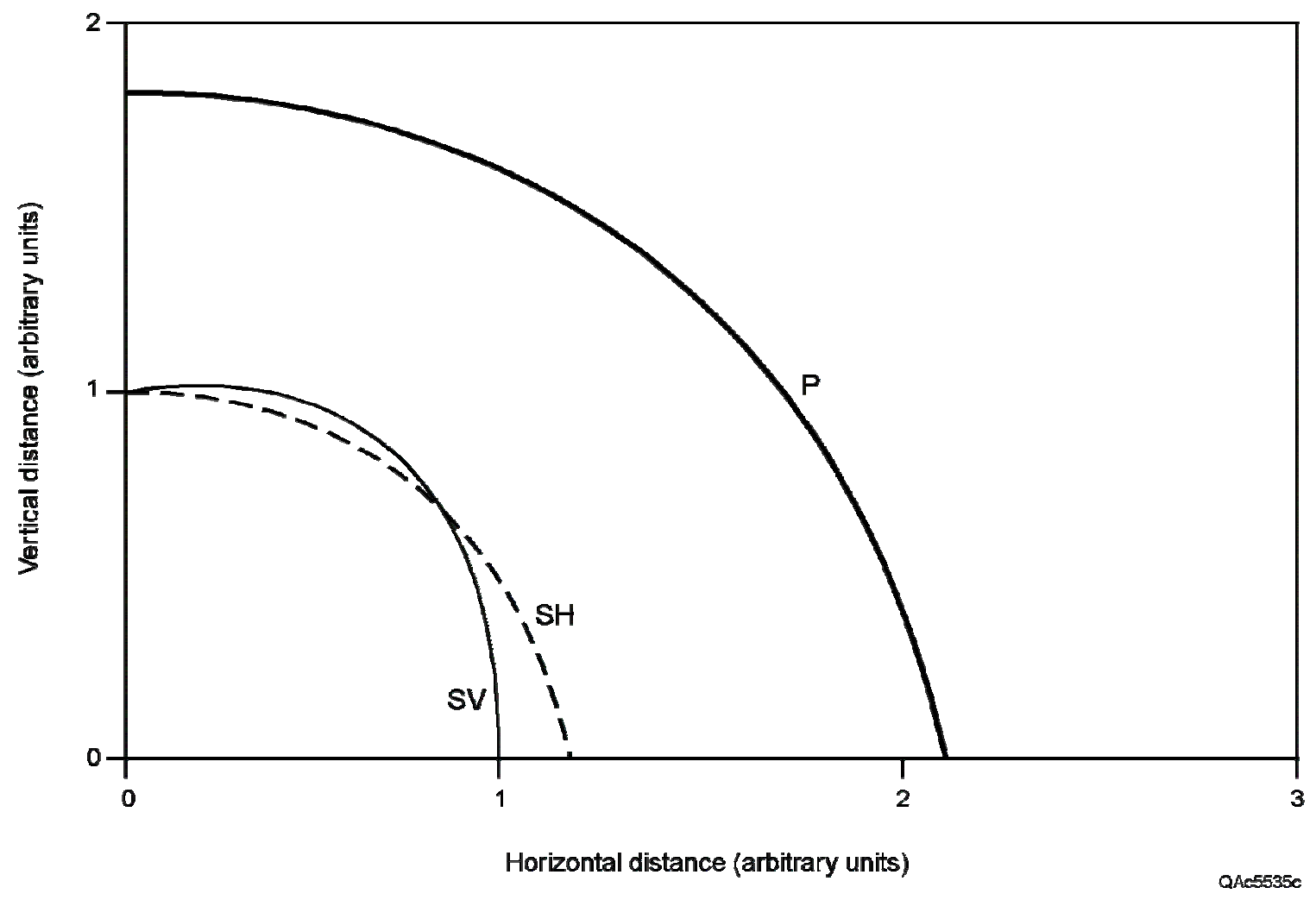

Figure 36. Wave mode velocities calculated for transverse isotropic (TI) media showing that 9-C SV and SH velocities differ in a flat-layered Earth (Levin, 1979; Levin, 1980). All wave modes propagate from a station positioned at the origin. The vertical axis represents downward, vertical propagation into the Earth. The horizontal axis represents propagation along the Earth’s surface. 
In summary, two key concepts are described by these Ashland data: SV-SV data are contaminated with SV-P data but SH-SH data are not, and SH velocity is different from SV velocity. These two fundamental distinctions sometimes cause one of the 9-C Swave modes, either SH-SH or SV-SV, to react to geologic conditions in a way different from that of the other mode. It is rarely apparent which mode, SH-SH or SV-SV, will provide more valuable information about a particular target. The best policy is to acquire data that allow both S-wave images to be created.

\section{3-C Data Polarization}

A key distinction between 9-C and 3-C S-wave data is that the polarization of the P-SV mode provided by 3-C technology is fundamentally different from the polarization of the SH-SH and SV-SV modes provided by 9-C technology. These distinctions are emphasized in the discussions of the 3-C and 9-C radiation patterns illustrated in Figures 21 through 25. We use data from a good-quality 3C3D seismic survey here to demonstrate that the preceding theoretical descriptions of P-SV polarization are correct.

For this demonstration, we select a source station at the center of the survey and then show the 3-C response observed at each of the four corners of the survey grid. Data acquired at receiver station 50 in the southeast corner of the survey and at receiver station 488 in the northwest corner are displayed in Figure 37. Because the azimuths from the source station to these two receiver stations differ by approximately $180^{\circ}$, the polarization of the P-SV data at these two receiver coordinates should differ by $180^{\circ}$. Positions of the source station and receiver station and orientations of the orthogonal inline and crossline vector sensors, $\mathrm{R}_{\mathrm{IL}}$ and $\mathrm{R}_{\mathrm{XL}}$, are shown in the map views of the 
seismic grid accompanying each data display. These horizontal vector sensors, $\mathrm{R}_{\mathrm{IL}}$ and $\mathrm{R}_{\mathrm{XL}}$, are deployed with the same orientation at every receiver station. Directions of the sensor arrows in the figure indicate the Earth-displacement directions that will produce positive responses for each sensor. The data trace labeled $\mathrm{V}$ is the response of the vertical vector sensor.
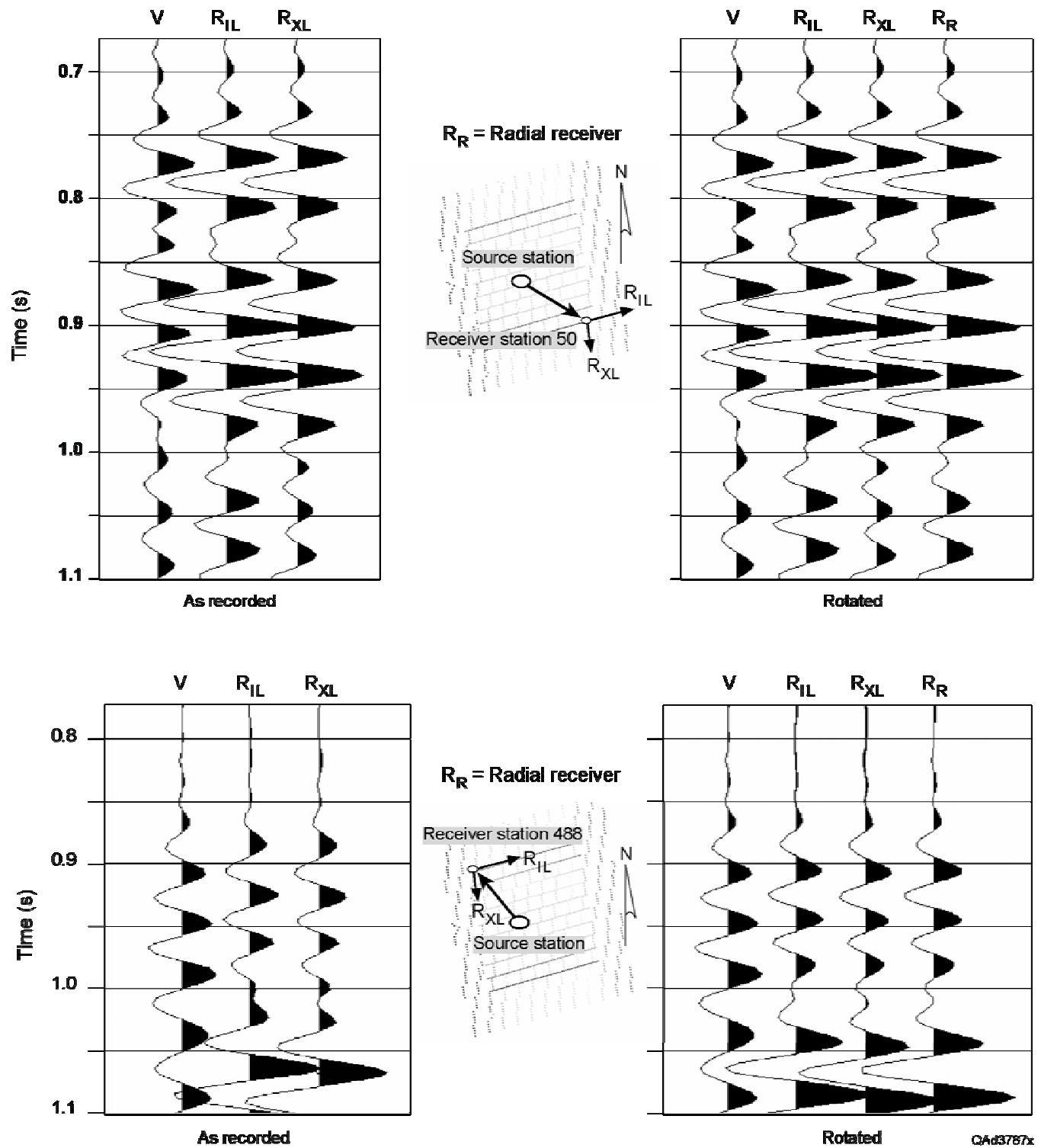

Figure 37. Example 1 of polarities of 3-C P-SV data measured in azimuth directions that differ by $180^{\circ}$. 
Raw, unprocessed 3-C data are shown in the panels on the left. Comparing the top and bottom panels shows that data recorded by orthogonal horizontal sensors at receiver station 50 have the opposite algebraic sign to that of the data recorded at receiver station 488. Thus the P-SV data exhibit a $180^{\circ}$ change in polarization for azimuth propagation directions that differ by $180^{\circ}$, as Figure 25 indicates. The data panels on the right show the P-SV data after processing steps have been taken to make the data appear to have been created by a 9-C horizontal-displacement source. For a horizontal-displacement source, there is no change in S-wave data polarity across the illuminated area (Fig. 25). That constant-polarity condition exists for the P-SV data in the right panels, and P-SV imaging can proceed only after this type of polarization correction has been made to all of the 3C3D data.

For completeness, data acquired in the other two corners of the 3C3D survey are shown in Figure 38. The principles illustrated here are the same as those that have been discussed for Figure 37. First, P-SV data acquired at receiver station 537 in the northeast corner have polarity opposite to the P-SV data acquired at receiver station 4 in the southwest corner (left panels). Second, the data can be altered to represent constantpolarization S-wave data similar to what would have been produced by a 9-C horizontaldisplacement source (right panels). The adjusted data in the right panels can now be used for image construction. 

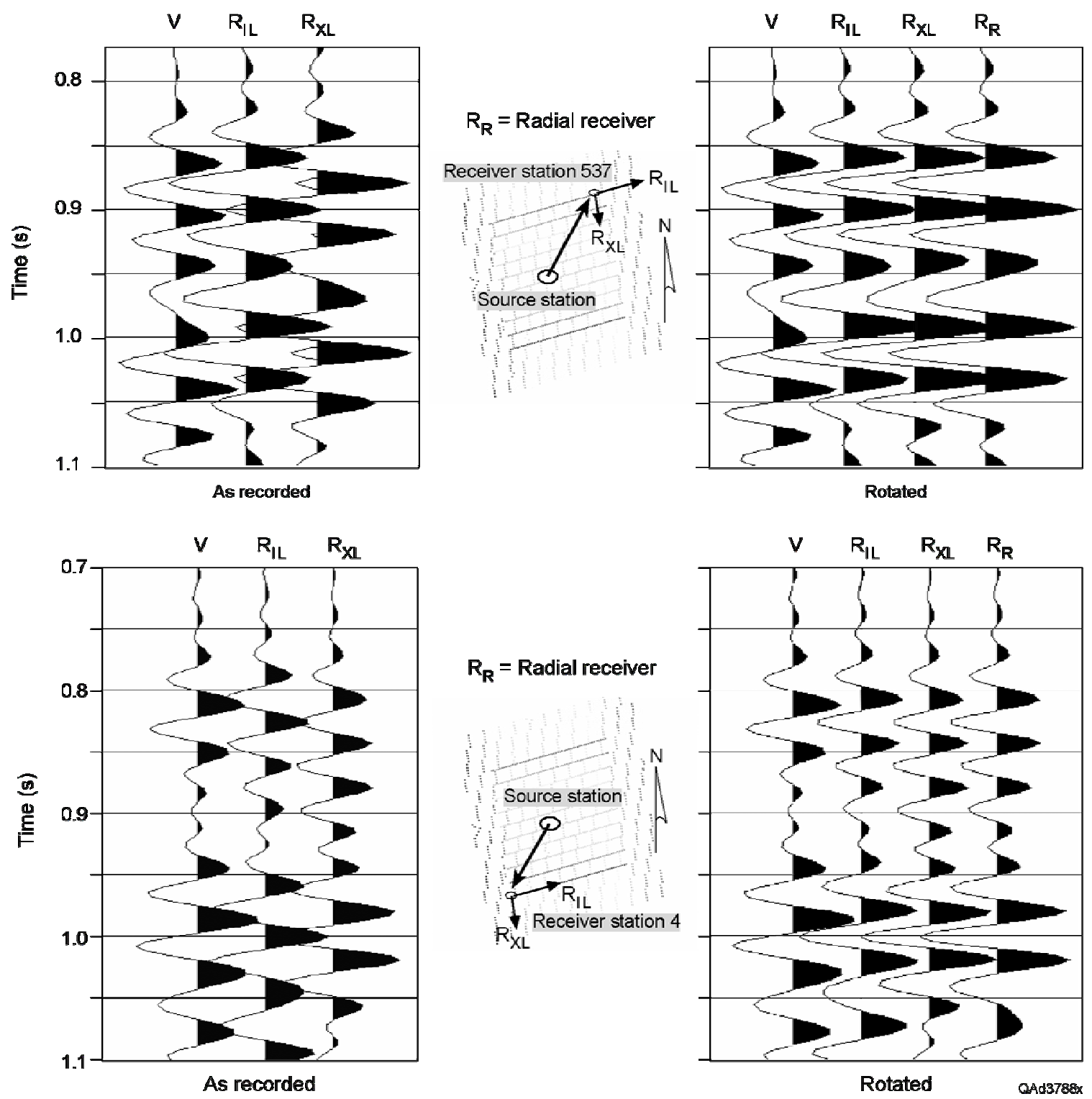

Figure 38. Example 2 of polarities of 3-C P-SV data measured in azimuth directions that differ by $180^{\circ}$.

This P-SV data polarization discussion has been expanded to cover a larger number of receiver stations by using an example from a second 3C3D seismic survey. These data are shown in Figure 39. The data in this figure are a 2-D profile extracted from the 3-D survey in which the receiver stations are inline with the source station. The source station is between receiver stations 269 and 270 at the center of the profile. The 
display documents response of the inline horizontal vector sensor along the profile. Data in the top panel confirm that inline horizontal displacements measured to the right of the source station (positive offset) have polarities that are opposite to the polarities of the horizontal displacements measured to the left of the source station (negative offset). This phenomenon is documented by comparing data polarities within data window A and by comparing data polarities between data windows B and C. The bottom panel shows the data after they have been polarity-adjusted to simulate 9-C horizontal-displacement source data. The adjusted data in this bottom panel can now be processed to create a PSV image. 

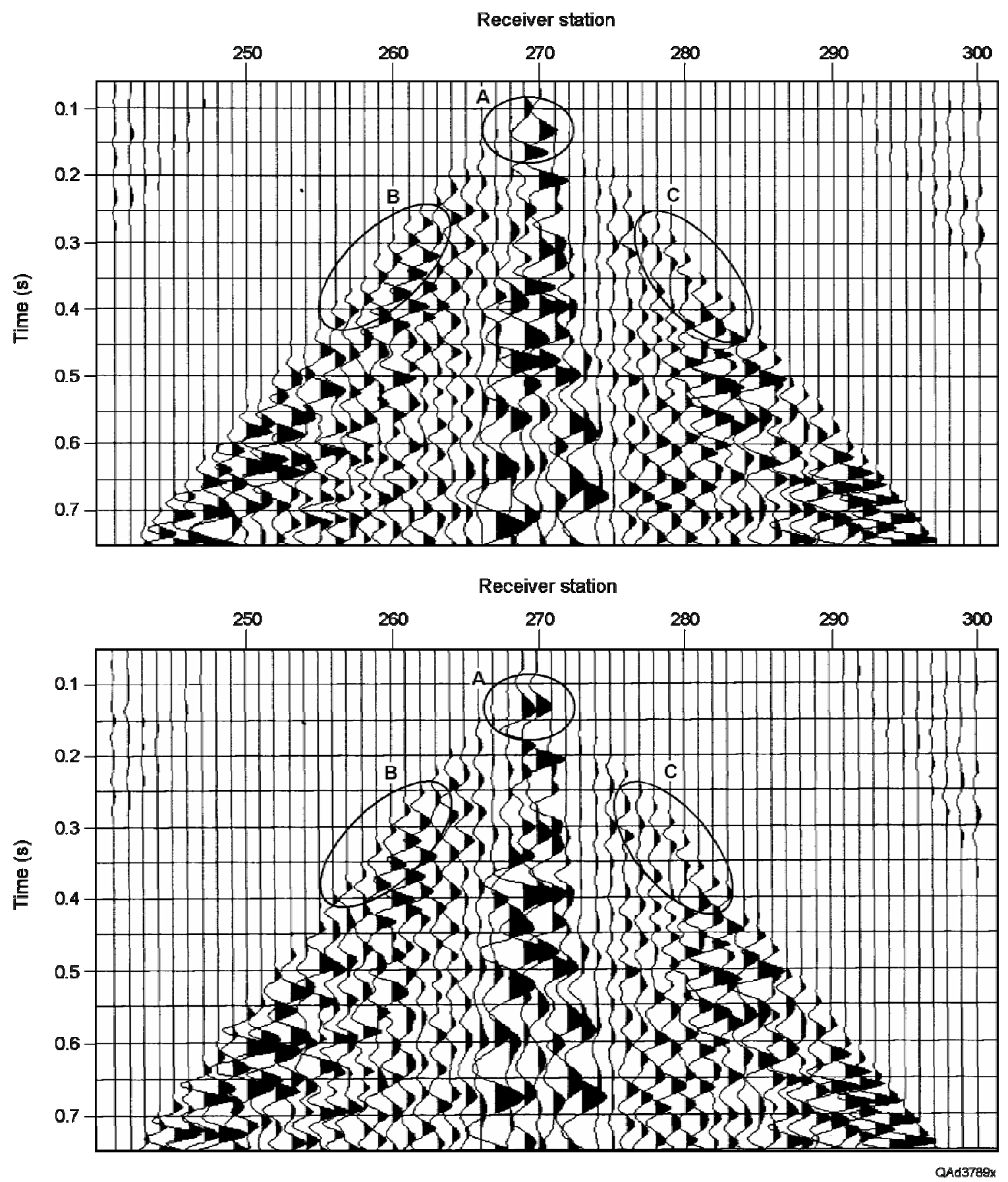

Figure 39. 2-D field record showing polarity behavior of the inline-horizontal component of P-SV data. 
Making 3-C and 9-C Images Depth Equivalent

When multicomponent seismic data are processed to make stacked and migrated images, S-wave images made from 9-C and 3-C data have different image-time axes because the two-way traveltime of a P-SV mode for a particular target depth is different from SH-SH and SV-SV traveltimes to and from that same depth coordinate. As a result, 9-C and 3-C S-wave modes position the reflection from a specific geologic interface at different traveltime coordinates. The principal problem that confronts interpreters who compare 9-C and 3-C S-wave data is the repeated dilemma of deciding which reflection events in each image are depth equivalent across the seismic image space.

Probably the most rigorous method for depth registering 9-C and 3-C S-wave data is to acquire and process multicomponent vertical seismic profile (VSP) data in a well inside the image space spanned by a multicomponent seismic survey. Because VSP data are acquired in the depth domain, each mode of a multicomponent VSP wavefield can be converted directly into either a depth-based image or a traveltime image (Hardage, 2000). An example of multicomponent VSP data being used to define depth-equivalent $\mathrm{SH}-\mathrm{SH}$ and SV-SV reflections was published by Hardage and others (2003). Depth registration techniques for 9-C and 3-C seismic data are also being developed that are internally selfconsistent within the multicomponent data themselves and do not have to rely on external calibration data such as a multicomponent VSP (Fomel and others, 2003). 


\section{CONCLUSIONS}

Multicomponent seismic technology cannot be properly understood or applied until all aspects of data generation, acquisition, processing, and interpretation are structured on vector-based principles. When vector-based thinking is used, it becomes more obvious why one S-wave mode may sense the Earth fabric differently than other Swave modes because the particle-displacement vector of each mode distorts the Earth in a different direction relative to the bedding planes, laminae, fractures, and other physical discontinuities that exist along the seismic propagation path. This concept that each Swave mode senses a different Earth fabric at a given subsurface coordinate is fundamental to understanding why all S-wave modes should be used in prospect evaluation rather than depending on only one S-wave mode to provide needed information.

Nine-component (9-C) seismic data provide three S-wave modes (SH-SH, SV$\mathrm{SV}$, and P-SV). Of these three modes, the SH-SH mode is the simplest in terms of its reflectivity behavior and its lack of contamination from other modes. In contrast, threecomponent (3-C) seismic data provide only one S-wave mode (P-SV). Each S-wave mode (SH-SH, SV-SV, and P-SV) involves a different subsurface illumination pattern, a different wavefield polarization, and a different reflectivity behavior. These distinctions mean that one S-wave mode may provide a critical piece of geologic information about a particular target that the other modes cannot. It is not possible to say that one S-wave mode will be more valuable than the other modes in all types of geological imaging problems. What can be said with confidence is that all information options are provided by 9-C seismic data because all S-wave modes can be extracted from such data. In 
situations where the SH-SH mode and/or the SV-SV mode (both being 9-C data) carries more valuable information than that of the P-SV mode (3-C data), it is unwise to restrict S-wave imaging to only 3-C technology.

In some cases, numerical modeling may indicate which S-wave mode will be the more valuable option for evaluating a particular geologic target. However, numerical modeling can sometimes be misleading. The safest course of action seems to be always to acquire 9-C seismic data if at all possible. In those cases where budget considerations and equipment constraints allow only 3-C data to be acquired, it is better to acquire 3-C data than to be content with 1-C P-wave data. 


\section{REFERENCES}

Aki, K., and Richards, P. G., 1980, Quantitative seismology-theory and methods: San Francisco, W. H. Freeman and Company, 557 p.

Fomel, S., Backus, M., DeAngelo, M., Murray, P., and Hardage, B., 2003, Multicomponent seismic data registration for subsurface characterization in the shallow Gulf of Mexico: Offshore Technology Conference, Houston.

Hardage, B., 2000, Vertical seismic profiling principles: seismic exploration series, third updated and revised edition: New York, Pergamon Press, v. 14, 552 p.

Hardage, B., DeAngelo, M., and Murray, P., 2003 Defining P-Wave and S-wave stratal surfaces with nine-component VSPs: The Leading Edge, v. 22, no. 8, p. 720-729.

Levin, F. K., 1979, Seismic velocities in transversely isotropic media I: Geophysics, v. 44, p. 918-936.

1980, Seismic velocities in transversely isotropic media, II: Geophysics, v. 45, p. 3-17.

Mitchum, R. M., Jr., 1977, Seismic stratigraphy and global changes of sea level, part 11, glossary of terms used in seismic stratigraphy, in Seismic stratigraphy-applications to hydrocarbon exploration: American Association of Petroleum Geologists, Memoir 26, p. 205-212.

Payton, C. E., 1977, Seismic stratigraphy-applications to hydrocarbon exploration: American Association of Petroleum Geologists, Memoir 26, 516 pages.

Simmons, J., Jr., Backus, M., Hardage, B., and Graebner, R., 1999, Case history: 3-D shear-wave processing and interpretation in radial-transverse (SV-SH) coordinates (exp. abs), in Technical Program, $69^{\text {th }}$ Annual International Meeting, Society of Exploration Geophysicists, v. 1, p. 792-795.

Simmons, J., Jr., and Backus, M., 1999, Radial-transverse (SV-SH) coordinates for 9-C $3-D$ seismic reflection data analysis (exp. abs), in Technical Program, $69^{\text {th }}$ Annual International Meeting, Society of Exploration Geophysicists, v. 1, p. 728-731.

White, J. E., 1983, Underground sound-applications of seismic waves: New York, Elsevier Science Publishers, 238 p. 